Pacific Journal of Mathematic 


\title{
REGULAR LATTICE MEASURES: MAPPINGS AND SPACES
}

\author{
George Bachman and Alan Sultan
}

\begin{abstract}
We prove in this paper a very general measure extension theorem which has as corollaries many recent, significant extension theorems in the literature. We apply these results to the question of when there is a well behaved map from the $\sigma$-smooth lattice regular measures on one set to the $\sigma$ smooth lattice regular measures on a second set. After developing these general theorems we specialize consideration to two valued latitce regular measures and obtain in a new and consistent manner many important mapping and subspace theorems on the preservation of different types of repleteness including results of Dykes, Hager, Isiwata, Moran, Varadarajan, Gillman, Jerrison and others.
\end{abstract}

Introduction. In earlier papers [6], [7], [49], we have developed for an abstract set $X$ and a given lattice $\mathscr{L}$ of subsets, the concept of $\mathscr{L}$-repleteness. This concept, as well as others such as $\mathscr{L}$ compact, $\mathscr{L}$-countably compact, etc., considered by Alexandroff [1-3], Meyer [41], Marczewski [39], Topsøe [50], Frolik [19], and others, we have expressed measure theoretically in terms of two valued $\mathscr{L}$-regular measures. This is advantageous; for besides being analytically simpler to work with than with filters, many theorems in this form can be generalized naturally to arbitrary $\mathscr{L}$-regular measures, and the topological settings extended from Wallman topologies to vague topologies.

The notion of $\mathscr{L}$-replete includes as special cases: real compact, Borel complete [24], $\alpha$-complete [15], etc., and in [6], we developed measure-theoretic results to show systematically how to obtain repleteness interrelations. Here, we are concerned with mapping and subspace problems. The mapping questions are of the type: Given $T: X \rightarrow Y$ which is well-behaved (see $\S 5$ for details) with respect to two lattices $\mathscr{L}_{1}, \mathscr{L}_{2}$ of subsets of $X$ and $Y$ respectively, when does $T$ induce a well-behaved mapping $T^{* *}: M R\left(\sigma, \mathscr{L}_{1}\right) \rightarrow M R\left(\sigma, \mathscr{L}_{2}\right)$, where, in general, $M R(\sigma, \mathscr{L})$ designates the $\sigma$-smooth $\mathscr{L}$-regular measures on a set $X$ with respect to a lattice, $\mathscr{L}$, of subsets? We develop the major results of this type in $\S 5$, and when applied to the special case of two-valued measures, we get as corollaries important subspace and mapping results of Frolik [21], Ishiwata [31], Dykes [15, 16], Moran [42], Hager [24], Mrowka [43], Varadarajan [51], Gordon [23], as well as classical results in realcompactness as 
can be found in Gillman-Jerrison [22]. Further subspace generalities pertaining to repleteness and applications are obtained systematically in $\S \S 6$ and 7.

In $\S \S 2$ and 3 , we give the relevant notation and background material. We prove in $\S 4$, a very general extension theorem for measures which has among its corollaries the important extension theorems of Hardy and Lacey [27] and Marik [40]. We also show how some of Frolik's work on almost realcompactness fits into this setting; although, we don't appeal to this for our development. In $\S \S 5$ and 6 , a number of general mapping theorems are proved, and applications are given to preservation of repleteness. In $\S \S 6$ and 7, we give general subspace results pertaining to repleteness, and apply these. We also show how the work of Harris [28] on extending maps to Wallman compactifications can be generalized and fits easily into this framework.

The general results developed here lead also to many new theorems and improvements of old ones concerning preservation of specific types of repleteness under mappings, inverse images, and with respect to subspaces, and unions; these further applications appear also in $\S \S 5,6$ and 7 .

Topological terms used are consistent with those of [33].

2. Background and notations. We introduce certain lattice definitions in this section and certain preliminary and background material which will be useful for the topological applications of our principle measure-theoretic results. The terminology in the literature is by no means standard: $\mathscr{L}$ will designate throughout a lattice of subsets of an abstract set $X$. We will always assume that $\phi$ and $X$ belong to $\mathscr{L}$. This in no way limits the generality of the results. For many purposes, it suffices just to assume that $\mathscr{L}$ is a semilattice. This will be indicated below. Finally, we note that the initial definitions and results could all be given for an abstract lattice embedded in a complete Boolean algebra; indeed, some of this has been done in the even more general setting of frames (see [14]). Even some of our general results could be formulated for certain abstract nonatomic lattices by utilizing Wallman type representations in place of the Stone-Loomis type representation as used by Sikorski [48] in defining a lattice-integral of the Olmstead, Carathéodory, Kappos type (see, e.g., [45], [10], [32]). Since this generalization is not at all difficult and since the major thrust of this paper is towards measure-theoretic constructs and a systematic application to certain categories of topological problems, we will give all of the definitions and results in a point set framework, for consistency and ease of expression. 
Definition. (1) $\mathscr{L}$ is a delta lattice if $\mathscr{L}$ is closed under countable intersections.

(2) $\mathscr{L}$ is a complemented if $A \in \mathscr{L}$ implies $A^{\prime} \in \mathscr{L}$, where $A^{\prime}$ designates the complement of $A$. $\mathscr{L}$ is complement generated if $A \in \mathscr{L}$ implies $A=\bigcap_{n=1}^{n=\infty} A_{n}^{\prime}$, where each $A_{n} \in \mathscr{L}$.

(3) If $f$ is a real valued function defined on $X, f$ is $\mathscr{L}$-continuous if $f^{-1}(C) \in \mathscr{L}$ for every closed set $C \subset R$. If $\mathscr{L}$ is a $\delta$-lattice, then clearly $f$ is $\mathscr{L}$-continuous if and only if $f^{-1}(-\infty, a]$ and $f^{-1}[b, \infty)$ belong to $\mathscr{L}$ for each $a, b \in R$. We designate by $C_{b}(\mathscr{L})$, all bounded $\mathscr{L}$-countinuous functions, and by $C(\mathscr{L})$ all $\mathscr{L}$-continuous functions.

(4) $\mathscr{L}$ is a separating or $T_{1}$ lattice if $x, y \in X . \quad x \neq y$, implies there exists an $A \in \mathscr{L}$ such that $x \in A$ and $y \notin A$.

(5) $\mathscr{L}$ is disjunctive if for any $A \in \mathscr{L}$, and $x \notin A$, there exists a $B \in \mathscr{L}$ such that $x \in B$ and $B \cap A=\varnothing$.

(6) $\mathscr{L}$ is normal if $A, B \in \mathscr{L}$ with $A \cap B=\varnothing$ implies there exist $C, D \in \mathscr{L}$ such that $A \subset C^{\prime}, B \subset D^{\prime}$, and $C^{\prime} \cap D^{\prime}=\varnothing$.

Our definitions, so far, are to a large extent consistent with those of Alexandroff [1] and Frolik [19]. We emphasize that $X$ is simply an abstract set and $\mathscr{L}$ a lattice of subsets. If $X$ should be a topological space, the lattice of closed sets is, for example, normal if the space is normal, the lattice of open sets is normal if and only if the space is extremally disconnected; the lattice of zero sets in any Tychonoff space is always normal; while the lattice of compact sets is in general not normal.

(7) The lattice of zero sets of functions in $C(\mathscr{L})$ is denoted by $Z(\mathscr{L})$.

(8) $\tau(\mathscr{L})$ designates the lattice of arbitrary intersections of elements of $\mathscr{L}$, and $\delta(\mathscr{L})$ designates the lattice of countable intersections of elements of $\mathscr{L}$.

(9) $\mathscr{A}(\mathscr{L}), \sigma(\mathscr{L}), \rho(\mathscr{L})$, and $s(\mathscr{L})$ designate respectively, the algebra generated by $\mathscr{L}, \sigma$-algebra generated by $\mathscr{L}$, the smallest class containing $\mathscr{L}$ together with countable unions and intersections, and the Souslin sets obtained from $\mathscr{L}$.

(10) A real valued measure $\mu$ defined on an algebra of sets will only be assume finitely additive. Those that are countably additive will be called $\sigma$-smooth. Those that are additive for arbitrary cardinal are called $\tau$-smooth.

We will not be concerned to any extent with $\tau$-smooth measures in this paper, mainly because we wish to apply our results to certain classes of topological problems; we will consider the $\tau$-smooth type applications as well as tight measures elsewhere. However, it should be pointed out that all our abstract extension and mapping theorems remain valid, as do the proofs, for the $\tau$-smooth measures; the only changes that are necessary, for example, is replacement in the 
definitions and relevant statements of theorems of countability by arbitrary cardinal.

(11) A measure $\mu$ defined on $\mathscr{A}(\mathscr{L})$ is $\mathscr{L}$-regular if for each $E \in \mathscr{A}(\mathscr{L})$ there exists an $A \in \mathscr{L}$ with $A \subset E$, and such that $|\mu(E)-\mu(A)|<\varepsilon$. Clearly, if $\mu$ is nonnegative, then $\mu$ is $\mathscr{L}$-regular if and only if $\mu(E)=\sup \mu(A), A \subset E, A \in \mathscr{L}$. If $\mu$ is an arbitrary $\mathscr{L}$-regular measure then it can be written as the difference of two nonnegative $\mathscr{L}$-regular measures. We refer the reader to Alexandroff [2] for further elementary properties of $\mathscr{L}$-regular measures, which will be utilized throughout, sometimes without explicit reference.

(12) $M(\mathscr{L})$ designates the set of all real valued measures on $\mathscr{A}(\mathscr{L}) . \quad M R(\mathscr{L})$ is the subset of $M(\mathscr{L})$ consisting of all the $\mathscr{L}$ regular measures. $M(\sigma, \mathscr{L})$ is the subset of $M(\mathscr{L})$ consisting of all the $\sigma$-smooth measures. $M R(\sigma, \mathscr{L})$ is the subset of $M(\sigma, \mathscr{L})$ consisting of all the $\sigma$-smooth $\mathscr{L}$-regular measures. Similarly we introduce $M R(\tau, \mathscr{L}) . \quad I(\mathscr{L}), I R(\mathscr{L}), I(\sigma, \mathscr{L}), I R(\sigma, \mathscr{L})$, are the subsets of the corresponding $M$ 's consisting of the nontrivial zeroone valued measures.

Finally, we denote by $I\left(\sigma^{*}, \mathscr{L}\right)$ the subset of $I(\mathscr{L})$ consisting of just those zero-one valued measures which are $\sigma$-smooth on $\mathscr{L}$ but not necessarily on all of $\mathscr{L}(\mathscr{L})$. We note that it does not follow that given a content on $\mathscr{L}$ which is $\sigma$-smooth that it can necessarily be extended to $\sigma(\mathscr{L})$. This question is related to tightness, details can be found in [46], [50], [34]. We do note that if $\mathscr{L}$ is a $\delta$-lattice then any $\sigma$-smooth $\mathscr{L}$ regular measure on $\mathscr{A}(\mathscr{L})$ can be extended to $\sigma(\mathscr{L})$ and will be $\mathscr{L}$-regular on all of $\sigma(\mathscr{L})$; use of this fact will be made in many proofs.

(13) $\mathscr{L}$ is called compact if $X=\bigcup A_{\alpha}^{\prime}$, where $A_{\alpha} \in \mathscr{L}$ implies that a finite number of the $A_{\alpha}^{\prime}$ cover $X$. Similarily we define $\mathscr{L}$ is countably compact, or $\mathscr{L}$ is Lindelof.

We note that the lattice of compact sets together with the entire space in a Hausdorff topological space is a compact lattice; the lattice of zero sets in a Tychonoff space is countably compact if and only if the space is pseudocompact. This follows by the well known Alexandroff-Glicksberg theorem (see, e.q. ([51], p. 170)). General properties and representation theorems for countably compact lattices can be found in [6].

(14) $\mathscr{L}$ is called regular if for $A \in \mathscr{L}$, and $x \notin A$, there exist $B, C \in \mathscr{L}$ such that $x \in B^{\prime}, A \subset C^{\prime}$, and $B^{\prime} \cap C^{\prime}=\varnothing$.

It is clear that we could pursue these matters, and define further analogous topological-type properties in terms of lattices and check various interrelations. This has been done in [19], in [35] in an abstract lattice theoretic setting, and in [14] for frames. We will 
make use of a few of the obvious simple relationships following:

THEOREM 2.1. If $\mathscr{L}$ is countably compact, then $M R(\mathscr{L})=$ $\operatorname{MR}(\sigma, \mathscr{L})$.

THEOREM 2.2. If $\mathscr{L}$ is Lindelof, then $M R(\sigma, \mathscr{L})=M R(\tau, \mathscr{L})$.

The first theorem appears in ([2], p. 590), while the second theorem is a straightforward generalization of a result of Varadarajan ([51] p. 175) for Tychonoff spaces; see also ([41] p. 50). In this connection we also note:

THEOREM 2.3. If $\mathscr{L}$ is a $\delta$-lattice and if $\sigma(\mathscr{L})=\rho(\mathscr{L})$ or if $\sigma(\mathscr{L}) \subset s(\mathscr{L}) ;$ then $M(\sigma, \mathscr{L})=M R(\sigma, \mathscr{L})$.

This theorem generalizes many specific topological type regularity theorems in the literature. For the details of the proof and applications see [6]. For the specific case of zero-one measures see [7].

(15) $\mathscr{L}$ is countably paracompact if $A_{n} \in \mathscr{L}, n=1,2, \cdots$, and $A_{n} \downarrow \varnothing$ imply there exist $B_{n} \in \mathscr{L}$ such that $A_{n} \subset B_{n}^{\prime}$ and $B_{n}^{\prime} \downarrow \varnothing$.

This is indeed equivalent to the usual topological characterization when $\mathscr{L}$ is the lattice of closed sets (see [5]). It is easy to see that if $\mathscr{L}$ is complement generated, then $\mathscr{L}$ is countably paracompact.

(16) If $X$ is a topological space we denote:

$\mathcal{O}=$ lattice of open sets

$\mathscr{F}=$ lattice of closed sets

$\mathscr{Z}=$ lattice of zero sets of continuous functions

$\mathscr{K}=$ lattice of compact sets, with $X$ adjoined.

Note: $\mathscr{K}$ is trivially still a compact lattice in the case of a Hausdorff space.

Our final set of definitions concern relationships between two lattices $\mathscr{L}_{1}, \mathscr{L}_{2}$ of subsets of $X$. We assume throughout that $\mathscr{L}_{1} \subset \mathscr{L}_{2}$; although this demand could be relaxed in a few of the subsequent results. This however is not important for our purposes.

(17) $\mathscr{L}_{1}$ semiseparates $\mathscr{L}_{2}$ if $A \in \mathscr{L}_{1}, B \in \mathscr{L}_{2}$ and $A \cap B=\varnothing$ implies there exists a $C \in \mathscr{L}_{1}$ such that $B \subset C$ and $A \cap C=\varnothing$.

$\mathscr{L}_{1}$ separates $\mathscr{L}_{2}$ if $A, B \in \mathscr{L}_{2}$ and $A \cap B=\varnothing$, then there exists $C, D \in \mathscr{L}_{1}$ such that $A \subset C, B \subset D$ and $C \cap D=\varnothing$.

$\mathscr{L}_{1}$ coseparates $\mathscr{L}_{2}$ if $A, B \in \mathscr{L}_{2}$ and $A \cap B=\varnothing$ implies there exists $C, D \in \mathscr{L}_{1}$ such that $A \subset C^{\prime}, B \subset D^{\prime}$ and $C^{\prime} \cap D^{\prime}=\varnothing$.

It is easy to prove: $\mathscr{L}_{1}$ coseparates $\mathscr{L}_{2}$ implies $\mathscr{L}_{1}$ separates $\mathscr{L}_{2}$ implies $\mathscr{L}_{1}$ semiseparates $\mathscr{L}_{2}$. For further details in abstract lattices see [13] and [35]. 
(18) Let $\mathscr{L}_{1} \subset \mathscr{L}_{2} . \quad \mathscr{L}_{2}$ is $\mathscr{L}_{1}$-countably paracompact if given $A_{n} \in \mathscr{L}_{2}, n=1,2,3, \cdots$ with $A_{n} \downarrow \varnothing$, there exists $B_{n} \in \mathscr{L}_{1}$, such that $A_{n} \subset B_{n}^{\prime}$ and $B_{n}^{\prime} \downarrow \varnothing$. It is easy to prove that if $\mathscr{L}_{2}$ is countably paracompact and if $\mathscr{L}_{1}$ separates $\mathscr{L}_{2}$, then $\mathscr{L}_{2}$ is $\mathscr{L}_{1}$-countably paracompact. We note that if $X$ is a normal countably paracompact topological space then $\mathscr{F}$ is clearly $\mathscr{Z}$-countably paracompact.

(19) Let $\mathscr{L}_{1} \subset \mathscr{L}_{2}$ be lattices of subsets of $X$. If $B_{n} \in \mathscr{L}_{2}$, $n=1,2,3, \cdots$ and $B_{n} \downarrow \varnothing$ implies there exists $A_{n} \in \mathscr{L}_{1}, n=1,2,3, \cdots$ with $B_{n} \subset A_{n}$ and $A_{n} \downarrow \varnothing$ then $\mathscr{L}_{2}$ is called a c.b $\mathscr{L}_{1}$ lattice.

If $X$ is a c.b space then $\mathscr{F}$ is a c.b $\mathscr{Z}$ lattice (see [5]). If $\mathscr{L}_{2}$ is a c.b $\mathscr{L}_{1}$ lattice and $\mathscr{L}_{1}$ is countably paracompact then $\mathscr{L}_{2}$ is $\mathscr{L}_{1}$ countably paracompact. Thus in a c.b topological space $\mathscr{F}$ is $\mathscr{Z}$ countably paracompact.

We next state for convenience, the fundamental representation theorem of Alexandroff.

TheOREM 2.4. Let $\mathscr{L}$ be a delta normal lattice. Then the conjugate space of $C_{b}(\mathscr{L})$ is $M R(\mathscr{L})$; i.e., to every bounded linear functional $\phi$ on $C_{b}(\mathscr{L})$ there exists a unique $\mu \in M R(\mathscr{L})$ such that $\phi(f)=\int f d \mu$, and $\|\phi\|=|\mu|$, the total variation of $\mu$. Moreover the positive and negative parts of $\phi$ correspond to the positive and negative parts of $\mu$. If $\phi \geqq 0$ and $A \in \mathscr{L}$ then $\mu(A)=\inf \phi(f)$, where $K_{A} \leqq f \leqq 1, f \in C_{b}(\mathscr{L}) K_{A}$ denotes the characteristic function of $A$.

$M R(\mathscr{L})$ can be topologized with the vague topology: a basic set of neighborhoods consisting of all

$$
\begin{aligned}
& V\left(\mu_{0}, f_{1}, \cdots, f_{n}, \varepsilon\right) \\
& \quad=\left\{\mu \in M R(\mathscr{L})_{i}\left|\int f_{1} d \mu-\int f: d \mu_{0}\right|<\varepsilon, \quad i=1,2, \cdots, n\right\}
\end{aligned}
$$

where $\mu_{0} \in M R(\mathscr{L})$ and the $f_{i} \in C_{b}(\mathscr{L})$. Using the Portmanteau Theorem in the case of a $\delta$-normal lattice $\mathscr{L},[3$, p. 180] it is easy to show that the restriction of this topology to $\operatorname{IR}(\mathscr{L})$ yields the Wallman-Frink topology on $\operatorname{IR}(\mathscr{L})$, having a base for the closed sets, all $W(A)=\{\mu \in I R(\mathscr{L}) \mid \mu(A)=1\}$ where $A \in \mathscr{L}$ (see [18], [52]). Its association with the filter approach will be indicated in the next section; it, of course, reduces in the case of abstract complemented lattices to the Stone representation space.

3. Topological lattices and zero-one measures. In this section, we will first summarize some simple measure and filter correspondences. The various zero-one measure characterizations of 
some of the earlier lattice topological notions will then be given, and finally some simple mappings between spaces of zero-one measures will be given. Some of the results of this section are known, and we collect them for the convenience of the reader.

We first summarize some results from [7].

THEOREM 3.1. There exists a 1-1 correspondence between all zero one $\mathscr{L}$-regular measures i.e., all elements of $\operatorname{IR}(\mathscr{L})$ and all $\mathscr{L}$-ultrafilters. There exists a 1-1 correspondence between $\operatorname{IR}(\sigma, \mathscr{L})$ and all $\mathscr{L}$-ultrafilters with the countable intersection property. In each case the correspondence is given by the following: With each $\mathscr{L}$-ultrafilter $T$ we associate the zero one measure $\mu_{T}$ defined on $\mathscr{A}(\mathscr{L})$ as follows: $\mu_{T}(E)=1$ if $E$ contains some $A \in T$ and $\mu_{T}(E)=0$ if $E$ is contained in the complement of some $A \in T$.

It is easy to see that there is also a 1-1 correspondence between prime $\mathscr{L}$-filters and all elements of $I(\mathscr{L})$. This correspondence is achieved by associating with each $\mu \in I(\mathscr{L})$ the prime filter given by $\{A \in \mathscr{L}: \mu(A)=1\}$. This correspondence induces a 1-1 correspondence between prime filters with the countable intersection property and $I\left(\sigma^{*}, \mathscr{L}\right)$. The details are easy.

If $\mathscr{C}$ is just a multiplicative system of subsets of $X$ (i.e. closed under finite intersections) then since $\mathscr{A}(\mathscr{C})=\mathscr{A}(\mathscr{L})$ where $\mathscr{L}$ is the generated lattice, and since $\mu \in I R(\mathscr{L})$ if and only if $\mu$ is regular with respect to $\mathscr{C}$ (obvious definition) we get that there is a 1-1 correspondence between prime (ultra) filters of $\mathscr{C}$ and prime (ultra) filters of $\mathscr{L}$.

Let $\mu \in I(\mathscr{L}) . \quad \mu$ is called fixed if $\bigcap\{A \in \mathscr{L}: \mu(A)=1\} \neq \varnothing$. The following results are known. They appear in [19] in a filter form and in [13] and [35] in an abstract lattice form.

(3.1) $\mathscr{L}$ is compact if and only if every $\mu \in I R(\mathscr{L})$ is fixed.

(3.2) If $\mathscr{L}$ is countably compact, then $\operatorname{IR}(\mathscr{L})=\operatorname{IR}(\sigma, \mathscr{L})$.

(3.3) $\mathscr{L}$ is normal if and only if for each $\mu \in I(\mathscr{L})$ there is a unique $\nu \in I R(\mathscr{L})$ such that $\mu \leqq \nu$ on $\mathscr{L}$.

(3.4) $\mathscr{L}$ is regular if and only if whenever $\mu_{1}, \mu_{2} \in I(\mathscr{L})$ and $\mu_{1} \leqq \mu_{2}$ on $\mathscr{L}$, then

$$
\bigcap\left\{A \in \mathscr{L} \mid \mu_{1}(A)=1\right\}=\bigcap\left\{A \in \mathscr{L} \mid \mu_{2}(A)=1\right\} .
$$

Definition 3.1. $X$ or $\mathscr{L}$ is called $\mathscr{L}$-replete if every $\mu \in I R(\sigma, \mathscr{L})$ is fixed.

If $\mathscr{L}$ is a disjunctive separating lattice this is equivalent to 
demanding that each $\mu \in \operatorname{IR}(\mathscr{L})$ is concentrated at a point; i.e., that $\operatorname{IR}(\sigma, \mathscr{L})$ is the set of all degenerate measures. Frolik [19] uses maximally complete, while some authors just use the word complete (see [4], [24]). In the case $\mathscr{L}$ is a separating, disjunctive delta lattice, $\operatorname{IR}(\sigma, \mathscr{L})$ with Wallman topology is called the $\mathscr{L}$-repletion of $X$. In this case, the mapping $x \rightarrow \mu_{x}$ (the two valued measure concentrated at $x$ ) is a dense embedding of $X$ in $\operatorname{IR}(\sigma, \mathscr{L})$. In the case when $\mathscr{L}$ is a strongly normal delta lattice i.e., $\mathscr{L}$ is a normal separating disjunctive delta lattice then every $f \in C(\mathscr{L})$ can be extended to an $\hat{f} \in C(I R(\sigma, \mathscr{L}))$ by defining $\hat{f}(\mu)=\int f d \mu$. The details can be found in [49]. If $X$ is a topological space and $\mathscr{L}$ is a separating disjunctive lattice which is a base for the closed sets $\tau(\mathscr{L})$ i.e., ( $\mathscr{L}$ is a basic, separating disjunctive lattice), then the map $x \rightarrow \mu_{x}$ also gives a homeomorphic mapping of $X$ into a dense subset of $\operatorname{IR}(\mathscr{L})$. For $X$ a Tychonoff space and $\mathscr{L}=\mathscr{X}, I R(\sigma, \mathscr{L})$ reduces to the ordinary realcompactification of Hewitt; and $\mathscr{Z}$-replete becomes realcompact (i.e., a $Q$-space) [30]). Further special cases and interrelations will be given in the next section.

Following Frolik [19], we define:

Definition 3.2. $X$ or $\mathscr{L}$ is $\mathscr{L}$-almost replete is every $\mu \in I R\left(\mathscr{L}^{\prime}\right) \cap I\left(\sigma^{*}, \mathscr{L}\right)$ is fixed, where $\mathscr{L}^{\prime}$ is the complementary lattice.

Frolik uses the expression comaximally complete. If $X$ is a topological space and if $\mathscr{L}=\mathscr{F}$, then $\mathscr{L}$-almost replete reduces to Frolik's original definition of almost real compact [see 21]. The following are extremely easy to prove:

(3.5) If $\mathscr{L}$ is regular then every $\mu \in I\left(\sigma^{*}, \mathscr{L}\right)$ is fixed if and only if $\mathscr{L}$ is $\mathscr{L}$-almost replete.

(3.6) If $\mathscr{L}$ is countably paracompact and normal and if every $\mu \in \operatorname{IR}(\sigma, \mathscr{L})$ is fixed then every $\mu \in I\left(\sigma^{*}, \mathscr{L}\right)$ is fixed.

The proof of (3.6) depends on the following simple fact:

(3.7) If $\mathscr{L}$ is normal and countably paracompact and if $\mu_{1}, \mu_{2} \in I(\mathscr{L})$ and $\mu_{1} \leqq \mu_{2}$ on $\mathscr{L}$, then $\mu_{1} \in I\left(\sigma^{*}, \mathscr{L}\right)$ implies $\mu_{2} \in I\left(\sigma^{*}, \mathscr{L}\right)$.

The following is a simple generalization of the well known relation between realcompact and almost realcompact.

THEOREM 3.2. (a) If $\mathscr{L}_{1} \subset \mathscr{L}_{2} \subset \tau\left(\mathscr{L}_{1}\right)$ and $\mathscr{L}_{1}$ is countably paracompact and normal, then $\mathscr{L}_{1}$-replete implies $\mathscr{L}_{2}$-almost replete. 
(b) If $\mathscr{L}_{1} \subset \mathscr{L}_{2}$, and $\mathscr{L}_{2}$ is regular, and $\mathscr{L}_{2}$ is $\mathscr{L}_{1}$-countably paracompact, then $\mathscr{L}_{2}$ almost replete implies $\mathscr{L}_{1}$-replete.

Proof. (a ) Let $\mu \in I R\left(\mathscr{L}_{2}^{\prime}\right) \cap I\left(\sigma^{*}, \mathscr{L}_{2}\right)$. By (3.3), there exists a $\nu \in \operatorname{IR}\left(\mathscr{L}_{1}\right)$ such that $\mu \leqq \nu$ on $\mathscr{L}_{1} ; \nu \in \operatorname{IR}\left(\sigma, \mathscr{L}_{1}\right)$ by (3.7). Now if $B \in \mathscr{L}_{2}$ and $\mu(B)=1$, then $B=\bigcap_{\alpha} A_{\alpha}, A_{\alpha} \in \mathscr{L}_{1}$ and $\nu\left(A_{\alpha}\right)=1$, all $\alpha$. As $\nu$ is fixed, the result is clear.

(b) Let $\mu \in I R\left(\sigma, \mathscr{L}_{1}\right)$. Then there exists a $\nu \in I R\left(\mathscr{L}_{2}\right)$ such that $\mu=\nu$ on $\mathscr{L}_{1}$. If $B_{n} \in \mathscr{L}_{2}, n=1,2,3, \cdots$, and $B_{n} \downarrow \varnothing$, then there exist $A_{n} \in \mathscr{L}_{1}, n=1,2,3, \cdots$, such that $B_{n} \subset A_{n}^{\prime}$ and $A_{n}^{\prime} \downarrow \varnothing$. Now $\nu\left(B_{n}\right) \leqq \nu\left(A_{n}^{\prime}\right) \leqq \mu\left(A_{n}^{\prime}\right) \downarrow 0$. Therefore $\nu \in I R\left(\sigma, \mathscr{L}_{2}\right)$. Since $\mathscr{L}_{2}$ is regular we are done by (3.5).

CoRollary 3.1 (Frolik [21]). If $X$ is a Tychonoff space, then $X$ realcompact implies $X$ almost realcompact, and if $X$ is also normal and countably paracompact, the converse holds.

Proof. Take $\mathscr{L}_{2}=\mathscr{F}$ and $\mathscr{L}_{1}=\mathscr{Z}$. As is well known [22] $\mathscr{Z}$ is normal, complement generated, and therefore countably paracompact. Thus almost realcompactness is immediate from the theorem.

For the converse use the fact noted earlier that if $X$ is normal and countably paracompact then $\mathscr{F}$ is $\mathscr{z}$-countably paracompact.

REMARK 3.1. We note both parts of Theorem 3.2 can be strengthened; for example, part (a) shows that any $\mu \in I\left(\sigma^{*}, \mathscr{L}_{2}\right)$ is fixed i.e., $X$ is prime complete, in the sense of Frolik.

The development here was strictly meant to show how some of Frolik's work fits into this framework, it is not critical for our development or for the subsequent applications.

Before continuing with the theoretical development of zero-one measures, we note some particular cases (for more details and examples see [6], [8]).

(a) For $X$ a Tychnonoff space, and $\mathscr{L}=\mathscr{Z}, I R(\mathscr{L})$, (with Wallman topology as usual) is of course $\beta X$, the Stone-Čech compactification; and $\operatorname{IR}(\sigma, \mathscr{L})=v X$, the Hewitt realcompactification.

(b) For $X$ a zero dimensional Hausdorff space and $\mathscr{L}=$ the lattice of clopen sets $\operatorname{IR}(\mathscr{L})=\beta_{0} X$, the Banaschewski compactification [9]; $\operatorname{IR}(\sigma, \mathscr{L})=\nu_{0} X$ is related to the $N$-compact spaces (see in particular [29], [12]).

(c) For $X$ a topological space, $\operatorname{IR}(F, O)$ where $\operatorname{IR}(F, \mathcal{O})=$ $\{\mu \in \operatorname{IR}(\mathscr{O}): \mu$ is not fixed $\}$ having the basic open sets: $0 \cup W(0)$ where $0 \in \mathcal{O}$ we get Katetov's absolute $X$ (see e.g., [17], [37], [38]) and with $\operatorname{IR}(\sigma, \mathcal{O})$ in place of $\operatorname{IR}(\mathcal{O})$ the $\alpha$-closure of Liu [38].

It is clear that (c) could be abstracted to a considerable degree, 
akin to Frolik's almost realcompactness. We do not pursue this here since it is somewhat removed from the major thrust of our applications.

We conclude this section with a consideration of extensions and restrictions of zero-one regular measures, and in the next section, we will consider the more difficult problem of arbitrary regular measures.

We assume throughout that $X$ is an abstract set and $\mathscr{L}_{1}, \mathscr{L}_{2}$ are, as usual, two lattices of subsets with $\mathscr{L}_{1} \subset \mathscr{L}_{2}$. It is clear, by Theorem 3.1, that any $\mu \in I R\left(\mathscr{L}_{1}\right)$ can be extended to a $\nu \in I R\left(\mathscr{L}_{2}\right)$. More precisely define for any $B \in \mathscr{L}_{2}, \nu(B)=1$ if there exists an $A \in \mathscr{L}_{1}$ such that $A \subset B$ and $\mu(A)=1$ and 0 otherwise. By Theorem 3.1, $\nu$ may be extended, and consequently viewed, as an element of $\operatorname{IR}\left(\mathscr{L}_{2}\right)$. We must just show that $\nu$ extends $\mu$. If $A \in \mathscr{L}_{1}$, and $\nu(A)=1$, and $\mu(A)=0$, then $\mu\left(A^{\prime}\right)=1$, and, since $\mu \in I R\left(\mathscr{L}_{1}\right)$, there exists a $B \subset \mathscr{L}_{1}, B \subset A^{\prime}$ and $\mu(B)=1$; whence, $\nu(B)=1$; but $\nu(A)=1$, a contradiction, so $\nu$ extends $\mu$.

Next we have

LEMma 3.1. If $\mathscr{L}_{1} \subset \mathscr{L}_{2}$ and $\mathscr{L}_{1}$ semiseparates $\mathscr{L}_{2}$ then for any $\nu \in I R\left(\mathscr{L}_{2}\right)$, the restriction, $\mu$, of $\nu$ to $\mathscr{A}\left(\mathscr{L}_{1}\right)$ belongs to $\operatorname{IR}\left(\mathscr{L}_{1}\right)$.

Proof. We must just show that $\mu$ is $\mathscr{L}_{1}$-regular. Thus suppose $A \in \mathscr{L}_{1}$ and $\mu(A)=\nu(A)=0$. Then $\nu\left(A^{\prime}\right)=1$, and there exists a $B \in \mathscr{L}_{2}$ such that $B \subset A^{\prime}$, and $\nu(B)=1$. By the semiseparation, there exists a $C \in \mathscr{L}_{1}$ with $B \subset C \subset A^{\prime}$, so $\mu(C)=1$. Thus $\mu$ is regular with respect to all $A^{\prime}$ with $A \in \mathscr{L}_{1}$, and consequently regular on all of $\mathscr{A}\left(\mathscr{L}_{1}\right)$.

We thus have that if $\mathscr{L}_{1}$ semiseparates $\mathscr{L}_{2}$ then the map $\phi: I R\left(\mathscr{L}_{2}\right) \rightarrow \operatorname{IR}\left(\mathscr{L}_{1}\right)$ given by restriction to $\mathscr{A}\left(\mathscr{L}_{1}\right)$ is a surjection.

Lemma 3.2. If $\mathscr{L}_{1} \subset \mathscr{L}_{2}$ and if $\mathscr{L}_{1}$ separates $\mathscr{L}_{2}$ then the restriction map $\phi: \operatorname{IR}\left(\mathscr{L}_{2}\right) \rightarrow \operatorname{IR}\left(\mathscr{L}_{1}\right)$ given by $\phi(\nu)$ is the restriction of $\nu$ to $\mathscr{A}\left(\mathscr{L}_{1}\right)$, is a bijection.

Proof. Let $\nu_{1}, \nu_{2} \in I R\left(\mathscr{L}_{2}\right)$, and $\nu_{1} \neq \nu_{2}$. Then there exist $A, B \in \mathscr{L}_{2}$ such that $\nu_{1}(A)=1, \nu_{2}(A)=0$, and $\nu_{1}(B)=0, \nu_{2}(B)=1$ and $A \cap B=\varnothing$. By the separation, there exists $C, D \in \mathscr{L}_{1}$ with $A \subset C, B \subset D$ and $C \cap D=\varnothing$ whence, $\nu_{1}(C)=1, \nu_{1}(D)=0$, and $\nu_{2}(D)=1, \nu_{2}(C)=0$, and we are done.

LEMma 3.3. If $\mathscr{L}_{1} \subset \mathscr{L}_{2}$ and if $\mathscr{L}_{1}$ semiseparates $\mathscr{L}_{2}$, then the restriction map $\phi: \operatorname{IR}\left(\mathscr{L}_{2}\right) \rightarrow \operatorname{IR}\left(\mathscr{L}_{1}\right)$ given by $\phi(\nu)$ is the restriction of $\nu$ to $\mathscr{A}\left(\mathscr{L}_{1}\right)$, is a bijection if and only if $\mathscr{L}_{1}$ separates $\mathscr{L}_{2}$. 
Proof. We must just prove the necessity of the condition. Thus, suppose the restriction is a bijection, but there are $A, B \in \mathscr{L}_{2}$ which cannot be separated by disjoint elements of $\mathscr{L}_{1}$. Then the set $S=\left\{C \in \mathscr{L}_{1} \mid A \subset C\right\} \cup\left\{D \in \mathscr{L}_{1} \mid B \subset D\right\}$, has the finite intersection property, and is contained in an $\mathscr{L}_{1}$-ultrafilter, with associated $\mu \in I R\left(\mathscr{L}_{1}\right)$. Let $E \in \mathscr{L}_{1}$, and $\mu(E)=1$. Then $E \cap A \neq \varnothing$; for if $E \cap A=\varnothing$, then, by semiseparation, there exists an $F \in \mathscr{L}_{1}$ such that $A \subset F$, and $E \cap F=\varnothing$, so $\mu(F)=0$. But since $A \subset F, \mu(F)=1$. This contradiction shows that $E \cap A \neq \varnothing$. Similarly, $E \cap B \neq \varnothing$, and clearly by Theorem 3.1 we can find $\nu_{1}, \nu_{2} \in I R\left(\mathscr{L}_{2}\right)$ both extending $\mu$ and with $\nu_{1}(A)=1=\nu_{2}(B)$, so $\nu_{1} \neq \nu_{2}$, a contradiction.

At this point we could already give a limited number of topological applications of these results but we will enlarge the framework considerably before giving applications. Further details along these lines and in an abstract lattice setting can be found in [13]. We summarize, for convenience of reference, the preceding results.

THEOREM 3.3. Let $\mathscr{L}_{1} \subset \mathscr{L}_{2}$ be two lattices of subsets of the abstract set $X$ such that $\mathscr{L}_{1}$ semiseparates $\mathscr{L}_{2}$. The restriction $\operatorname{map} \phi: \operatorname{IR}\left(\mathscr{L}_{2}\right) \rightarrow \operatorname{IR}\left(\mathscr{L}_{1}\right)$ given by $\phi(\nu)$ is the restriction of $\nu$ to $\mathscr{L}\left(\mathscr{L}_{1}\right)$ is a surjection, and is a bijection if and only if $\mathscr{L}_{1}$ separates $\mathscr{L}_{2}$.

We now consider related matters pertaining to the regular $\sigma$ smooth measures. If, as before, $\mathscr{L}_{1} \subset \mathscr{L}_{2}$ and $\mu \in I R\left(\sigma, \mathscr{L}_{1}\right)$. We know that there exist $\mathscr{L}_{2}$-regular measures extending $\mu$. If, $\nu \in I R\left(\mathscr{L}_{2}\right)$ and $\nu$ extends $\mu, \nu$ need not be $\sigma$-smooth. If however, $\mathscr{L}_{2}$ is $\mathscr{L}_{1}$ countably paracompact then for any sequence $B_{n} \in \mathscr{L}_{2}, n=1,2, \cdots$ with $B_{n} \downarrow \varnothing$, there exists $A_{n} \in \mathscr{L}_{1}, n=1,2, \cdots$ such that $B_{n} \subset A_{n}^{\prime}$, and $A_{n}^{\prime} \downarrow \varnothing$. Then $\nu\left(B_{n}\right) \leqq \mu\left(A_{n}^{\prime}\right) \downarrow 0$, since $\mu \in I R\left(\sigma, \mathscr{L}_{1}\right)$. Similarly if $\mathscr{L}_{2}$ is a c.b $\mathscr{L}_{1}$ lattice we have $\nu \in \operatorname{IR}\left(\sigma, \mathscr{L}_{2}\right)$. Combining this with the previous work, we have

TheOREM 3.4. If $\mathscr{L}_{1} \subset \mathscr{L}_{2}$ are two lattices of subsets of $X$, then

(a) Any $\mu \in I R\left(\mathscr{L}_{1}\right)$ can be extended to a $\nu \in I R\left(\mathscr{L}_{2}\right)$ and if $\mathscr{L}_{2}$ is $\mathscr{L}_{1}$ countably paracompact or $\mathscr{L}_{2}$ is countably compact, or a c.b $\mathscr{L}_{1}$ lattice, then any $\mathscr{L}_{2}$-regular extension of a $\mu \in I R\left(\sigma, \mathscr{L}_{1}\right)$ belongs to $\operatorname{IR}\left(\sigma, \mathscr{L}_{2}\right)$.

(b) If $\mathscr{L}_{1}$ semiseparates $\mathscr{L}_{2}$ and $\mathscr{L}_{2}$ is $\mathscr{L}_{1}$ countably paracompact or a c.b $\mathscr{L}_{1}$ lattice the restriction $\operatorname{map} \phi^{*}: \operatorname{IR}\left(\sigma, \mathscr{L}_{2}\right) \rightarrow$ $\operatorname{IR}\left(\sigma, \mathscr{L}_{1}\right)$ is a surjection.

(c) If $\mathscr{L}_{1}$ separates $\mathscr{L}_{2}$ and $\mathscr{L}_{2}$ is $\mathscr{L}_{1}$ countably paracompact, or a c.b $\mathscr{L}_{1}$ lattice, then the restriction map $\phi^{*}: \operatorname{IR}\left(\sigma, \mathscr{L}_{2}\right) \rightarrow \operatorname{IR}\left(\sigma, \mathscr{L}_{1}\right)$ is a bijection. 
CoRollary 3.2. If $\mathscr{L}_{1} \subset \mathscr{L}_{2}$ are two lattices of subsets of $X$, and $\mathscr{L}_{1}$ separates $\mathscr{L}_{2}$, and if $\mathscr{L}_{2}$ is countably paracompact, then the restriction map: $\phi^{*}: \operatorname{IR}\left(\sigma, \mathscr{L}_{2}\right) \rightarrow I R\left(\sigma, \mathscr{L}_{1}\right)$ is a bijection.

Proof. The hypotheses imply that $\mathscr{L}_{2}$ is $\mathscr{L}_{1}$ countably paracompact, as noted earlier.

In [6], we have systematically applied these results to topological repletion matters, and will give several applications in the next section; we will just give those which are particularly relevant to the mapping and subspace problems that we are concerned with in this paper. In addition to the statements in the above theorems, we can make statements pertaining to the continuity of the maps with respect to the Wallman topologies, however, we will not need this here (see, e.g., [6], [13], [35]).

4. General extensions and applications. Again, we consider the case of two lattices of sets $\mathscr{L}_{1}, \mathscr{L}_{2}$ with $\mathscr{L}_{1} \subset \mathscr{L}_{2}$, and both contained in the abstract set $X$. We now generalize the considerations of the previous section to the more difficult problem of extending a $\mu \in M R\left(\mathscr{L}_{1}\right)$ or $M R\left(\sigma, \mathscr{L}_{1}\right)$ to one in $M R\left(\mathscr{L}_{2}\right)$ or $M R\left(\sigma, \mathscr{L}_{2}\right)$, as well as the restriction matters. Problems of this type appear in many specific topological frameworks throughout the literature (see [27], [40], [36], [47]). We will develop briefly here a general extension theorem which covers many special cases in the literature. We will show the relationship of this to our previous considerations, and then we will give a few applications. In the remaining sections we will develop mapping properties in the general setting, and apply these results systematically, in the topological case to get many separate and diverse mapping and subspace results.

We first consider restriction question:

TheOREM 4.1. Let $\mathscr{L}_{1} \subset \mathscr{L}_{2}$ be two lattices of subsets of $X$. If (a) $\mathscr{L}_{1}$ semiseparates $\mathscr{L}_{2}$ then the restriction of $\mu \in M R\left(\mathscr{L}_{2}\right)$ $\left(M R\left(\sigma, \mathscr{L}_{2}\right)\right)$ to $\mathscr{A}\left(\mathscr{L}_{1}\right)$ belongs to $M R\left(\mathscr{L}_{1}\right)\left(M R\left(\sigma, \mathscr{L}_{1}\right)\right)$.

(b) If $\mathscr{L}_{1}$ is a $\delta$-lattice and if $\sigma\left(\mathscr{L}_{1}\right)=\rho\left(\mathscr{L}_{1}\right)$ or if $\sigma\left(\mathscr{L}_{1}\right) \subset$ $s\left(\mathscr{L}_{1}\right)$ then the restriction of any $\mu \in M R\left(\sigma, \mathscr{L}_{2}\right)$ to $\mathscr{A}\left(\mathscr{L}_{1}\right)$ belongs to $\operatorname{MR}\left(\sigma, \mathscr{L}_{1}\right)$.

Proof. Since the proof of (b) is a direct consequence of Theorem 2.3, we need only prove (a):

Proof of (a). We may assume $\mu \geqq 0$. Call the restriction of $\mu$ to $\mathscr{A}\left(\mathscr{L}_{1}\right)$, $\nu$. Suppose $E \in \mathscr{A}\left(\mathscr{L}_{1}\right)$. Then $E$ may be written as $E=\bigcup_{1}^{n} A_{i}-B_{i}$ where the union is disjoint and $B_{i} \subset A_{i}$. Since $\nu$ is 
$\mathscr{L}_{2}$ regular there exist $B_{i}^{*} \in \mathscr{L}_{2}, i=1,2, \cdots, n$, such that $B_{i}^{*} \subset A_{i}-B_{i}$ and $\nu\left(A_{i}-B_{i}\right)<\nu\left(B_{i}^{*}\right)+\varepsilon / 2^{i}$. By semiseparation, we may find $C_{i} \in \mathscr{L}_{1}$ such that $B_{i}^{*} \subset C_{i}$ and $C_{i} \cap B_{i}=\varnothing$ for each $i=1,2, \cdots, n$. Clearly $A_{i}-B_{i} \supset A_{i} \cap C_{i} \supset B_{i}^{*}$ for each $i$ and $\bigcup_{1}^{n} A_{i} \cap C_{i} \in \mathscr{L}_{1}$. We then have

$$
\begin{aligned}
\nu\left(\bigcup\left(A_{i} \cap C_{i}\right)\right) & =\sum \nu\left(A_{i} \cap C_{i}\right) \leqq \nu(E)=\sum \nu\left(A_{i}-B_{i}\right) \\
& <\left(\sum \nu\left(B_{i}^{*}\right)\right)+\varepsilon \leqq \sum \nu\left(A_{i} \cap C_{i}\right)+\varepsilon .
\end{aligned}
$$

Thus $\nu$ is $\mathscr{L}_{1}$ regular.

Now we turn to the extension problem, we consider this in two parts.

THEOREM 4.2. Let $\mathscr{L}_{1} \subset \mathscr{L}_{2}$ be two delta normal lattices of subsets of $X$ such that $\mathscr{L}_{1}$ semiseparates $\mathscr{L}_{2}$. Then any $\mu \in M R\left(\mathscr{L}_{1}\right)$ can be extended to a $\nu \in M R\left(\mathscr{L}_{2}\right)$. Moreover, the extension is always a unique extension if and only if $\mathscr{L}_{1}$ separates $\mathscr{L}_{2}$.

Proof. Again we may assume $\mu \geqq 0$. Form the linear functional $\Phi$ defined on $C_{b}\left(\mathscr{L}_{1}\right)$ as follows: $\Phi(f)=\int f d \mu$ for each $f \in C_{b}\left(\mathscr{L}_{1}\right)$. By Krein's theorem, $\Phi$ may be extended to a positive linear functional $\Phi^{*}$ on $C_{b}\left(\mathscr{L}_{2}\right)$. Since $\Phi^{*}$ is bounded, we may find by Theorem 2.4 a $\nu \in M R\left(\mathscr{L}_{2}\right)$ such that $\Phi^{*}(g)=\int g d \nu$ for each $g \in C_{b}\left(\mathscr{L}_{2}\right)$. Clearly $\nu(A) \leqq \mu(A)$ for $A \in \mathscr{L}_{1}$ since we are taking an infimum over a larger set. To show the inequality the other way we have since $\nu \in M R\left(\mathscr{L}_{2}\right)$, a $B \in \mathscr{L}_{2}$ such that $A \subset B^{\prime}, \nu\left(B^{\prime}\right)-\nu(A)<\varepsilon$. By semiseparation we can find a $C \in \mathscr{L}_{1}$ with $B \subset C$ and $A \cap C=\varnothing$. Since $\mathscr{L}_{1}$ is normal there exists an $f \in C_{b}\left(\mathscr{L}_{1}\right)$ such that $f(A)=1$ and $f(C)=1$ (see [1], p. 317). We have

$$
\mu(A) \leqq \int f d \mu=\int f d \nu=\int_{A} f d \nu+\int_{C^{\prime}-A} f d \nu+\int_{C} f d \nu<\nu(A)+\varepsilon .
$$

Since $\varepsilon$ was arbitrary we have $\mu(A) \leqq \nu(A)$ and therefore $\mu=\nu$ on $\mathscr{L}_{1}$. By Theorem 4.1, $\nu$ restricted to $\mathscr{A}\left(\mathscr{L}_{1}\right)$ is $\mathscr{L}_{1}$ regular and since $\mu=\nu$ on $\mathscr{L}_{1}, \mu=\nu$ on $\mathscr{L}\left(\mathscr{L}_{1}\right)$. Thus $\nu$ is an extension of $\mu$.

To complete the proof we must only show that there is a unique extension if and only if $\mathscr{L}_{1}$ separates $\mathscr{L}_{2}$. The necessity of the condition follows from Lemma 3.3.

To show the sufficiency, suppose $\mu \in M R\left(\mathscr{L}_{1}\right)$ has 2 distinct extensions $\nu_{1}$ and $\nu_{2}$ to $M R\left(\mathscr{L}_{2}\right)$. Then there is an $A \in \mathscr{L}_{2}$ such that $\nu_{1}(A) \neq \nu_{2}(A)$. We may suppose $\nu_{2}(A)-\nu_{1}(A)=\delta>0$. Choose $\varepsilon<\delta$. By $\mathscr{L}_{2}$ regularity there is a $B \in \mathscr{L}_{2}$ such that $\nu_{1}\left(B^{\prime}\right)-\nu_{1}(A)<\varepsilon$. By separation there exists $C, D \in \mathscr{L}_{1}$ such that $A \subset C, B \subset D$ and $C \cap D=\varnothing$. It follows that 


$$
\begin{aligned}
\delta=\nu_{2}(A)-\nu_{1}(A) & \leqq \nu_{2}(C)-\nu_{1}(A) \leqq \nu_{1}\left(D^{\prime}\right)-\nu_{1}(A) \leqq \nu_{1}\left(B^{\prime}\right)-\nu_{1}(A) \\
& <\varepsilon, \quad \text { a contradiction. }
\end{aligned}
$$

Thus each $\mu \in M R\left(\mathscr{L}_{1}\right)$ has a unique extension if $\mathscr{L}_{1}$ separates $\mathscr{L}_{2}$ and we are done.

THEOREM 4.3. Let $\mathscr{L}_{1} \subset \mathscr{L}_{2}$ be two delta normal lattices of subsets of $X$ such that $\mathscr{L}_{1}$ semiseparates $\mathscr{L}_{2}$ and such that (a) $\mathscr{L}_{2}$ is $\mathscr{L}_{1}$ countably paracompact or (b) $\mathscr{L}_{2}$ is a c.b $\mathscr{L}_{1}$ lattice or (c) $\mathscr{L}_{2}$ is countably compact. Then every $\mu \in M R\left(\sigma, \mathscr{L}_{1}\right)$ can be extended to a $\nu \in M R\left(\sigma, \mathscr{L}_{2}\right)$. The extension is always unique if $\mathscr{L}_{1}$ separates $\mathscr{L}_{2}$.

Proof. We need only show that the extension of a $\mu \in M R\left(\sigma, \mathscr{L}_{1}\right)$ is $\sigma$-smooth. If $\mathscr{L}_{2}$ is $\mathscr{L}_{1}$ countably paracompact, or $\mathscr{L}_{2}$ is a c.b $\mathscr{L}_{1}$ lattice, then the proof is similar to the proof of Theorem 3.4. If $\mathscr{L}_{2}$ is countably compact the result follows from Theorem 2.1.

We will give a few applications of these results. The first application is a slight strengthening of the main result of Hardy and Lacey [27].

Corollary 4.1. Let $\mathcal{O}_{1}$ and $\mathcal{O}_{2}$ be topologies on $X$ with closed sets $\mathscr{F}_{1}$ and $\mathscr{F}_{2}$ respectively. If $\mathscr{O}_{2}$ is a compact Hausdorff topology and $\mathscr{O}_{1}$ a regular topology with $\mathscr{O}_{1} \subset \mathcal{O}_{2}$, then every $\mu \in M R\left(\sigma, \mathscr{F}_{1}\right)$ $\left(=M R\left(\mathscr{F}_{1}\right)\right)$ can be extended to a $\nu \in M R\left(\sigma, \mathscr{F}_{2}\right)\left(=M R\left(\mathscr{F}_{2}\right)\right)$. The extension is unique if and only if $\mathscr{O}_{1}=\mathscr{O}_{2}$.

Proof. $\mathscr{F}_{1} \subset \mathscr{F}_{2}$ and both are delta normal, compact lattices. Moreover, $\mathscr{F}_{1}$ semiseparates $\mathscr{F}_{2}$ since in a regular space a compact set and a closed set disjoint from it can be separated by disjoint open sets. The desired extension follows from Theorem 3.1. The remainder is simple.

As a further generalization of Corollary 4.1 we have

CoRollary 4.2. If $\mathscr{O}_{1} \subset \mathcal{O}_{2}$ are two normal topologies for $X$ with closed sets $\mathscr{F}_{1}$ and $\mathscr{F}_{2}$ respectively such that $\mathscr{F}_{1}$ semiseparates $\mathscr{F}_{2}$ then if

(a) $\left(X, \mathcal{O}_{2}\right)$ is countably compact, or if

(b) $\left(X, \mathscr{O}_{2}\right)$ is countably paracompact and $\mathscr{F}_{1}$ separates $\mathscr{F}_{2}$, or if

(c) $\mathscr{F}_{2}$ is $\mathscr{F}_{1}$ countably paracompact, or a c.b $\mathscr{F}_{1}$ lattice then any $\mu \in M R\left(\sigma, \mathscr{F}_{1}\right)$ can be extended to $a \nu \in M R\left(\sigma, \mathscr{F}_{2}\right)$ and in case (b) the extension is unique. 
The following corollary is a strengthened version of the difficult portion of the extension theorem of Marik ([40]).

COROLlaRY 4.3. Let $X$ be a topological space which is countably paracompact and normal, then every $\mu \in M(\sigma, \mathscr{Z})$ extends uniquely to $a \nu \in M R(\sigma, \mathscr{F})$.

Proof. $\mathscr{Z} \subset \mathscr{F}$ and both are delta normal lattices. Since $\mathscr{Z}$ is complement generated $\sigma(\not \mathscr{Z})=\rho(\not \mathscr{Z})$ and therefore $M(\sigma, \mathscr{Z})=$ $M R(\sigma, \mathscr{Z})$. Moreover, $\mathscr{Z}$ coseparates $\mathscr{F}$ since $X$ is normal. It follows that $\mathscr{F}$ is $\mathscr{Z}$ countably paracompact and we are done by Theorem 4.3.

As a further simple application we have:

CoRollary 4.4. $\mathscr{O}_{1} \subset \mathcal{O}_{2}$ are two Tychonoff topologies for $X$ with zero sets $\mathscr{Z}_{1}$ and $\mathscr{Z}_{2}$ respectively such that $\mathscr{Z}_{1}$ semiseparates $\mathscr{X}_{2}$, then if

(a) $\left(X, \mathscr{O}_{2}\right)$ is pseudocompact, or if

(b) $\check{\varkappa}_{1}$ separates $\check{\varkappa}_{2}$, or if

(c) $\mathscr{\varkappa}_{2}$ is $\mathscr{\varkappa}_{1}$ countably paracompact, or a c.b 芩 lattice then any $\mu \in M\left(\sigma, \mathscr{Z}_{1}\right) \quad\left(=M R\left(\sigma, \mathscr{Z}_{1}\right)\right)$ can be extended to a $\nu \in M\left(\sigma, \mathscr{Z}_{2}\right)$ $\left(=M R\left(\sigma, \mathscr{Z}_{2}\right)\right)$ and in (b) the extension is unique.

Proof. We need only note that in (a), $\left(X, \mathscr{O}_{2}\right)$ pseudocompact is equivalent to being $\mathscr{Z}_{2}$-countably compact and in (b), $\mathscr{Z}_{2}$ is $\mathscr{Z}_{1}$ countably paracompact. (c) is clear.

We now give some general repleteness applications.

(4.1) $\sigma(\mathscr{L})$ replete implies $\mathscr{L}$-replete. In particular in a topological space $\sigma(\mathscr{F})$ replete (i.e., Borel replete) implies $\mathscr{F}$-replete.

\section{Proof. Clear.}

(4.2) If $X$ is a Tychonoff space, then $\mathscr{2}$-repletes implies $\mathscr{F}$ replete.

Proof. Let $\mu \in \operatorname{IR}(\sigma, \mathscr{F})$. Then the restriction $\nu$ of $\mu$ to $\sigma(\mathscr{Z})$ is $\sigma$-smooth and since $\sigma(\tilde{\Sigma})=\rho(\mathscr{z})$ we have by Theorem 2.3 that $\nu \in \operatorname{IR}(\sigma, \mathscr{\gamma})$. Therefore $\nu$ and hence $\mu$ is concentrated at a point.

(4.3) If $X$ is a Tychonoff space, then $\mathscr{Z}$-replete is equivalent to $\sigma(\mathscr{z})$-replete.

Proof. Since $\sigma(\mathscr{Z})=\rho(\mathscr{Z})$ we again have by Theorem 2.3 that $I R(\sigma, \mathscr{z})=I(\sigma, \mathscr{\not})$ and the proof is immediate.

(4.4) If $X$ is a Tychonoff space and if $X$ is normal and countably paracompact, then $\mathscr{F}$-replete implies $\mathscr{z}$-replete. 
Proof. According to Theorem 3.4 there is a 1-1 correspondence between $\operatorname{IR}(\sigma, \mathscr{F})$ and $\operatorname{IR}(\sigma, \mathscr{Z})$. The proof is now immediate.

This result was first proved by Dykes ([15], Corollary 1.10). If $X$ is a Tychonoff c.b space, $\mathscr{F}$ is $\mathscr{Z}$-countably paracompact and as in (4.4) $\mathscr{F}$-replete implies $\mathscr{Z}$-replete.

(4.5) If $\mathscr{L}$ is a delta lattice and if $\sigma(\mathscr{L}) \subset s(\mathscr{L})$ then $\mathscr{L}$-replete is equivalent to $\sigma(\mathscr{L})$-replete.

Proof. The proof is analogous to (4.3).

REMARK 4.1. For $X$ an analytic space (the continuous image of a Polish space) $\sigma(\mathscr{F}) \subset s(\mathscr{F})$. Thus is an analytic space $\sigma(\mathscr{F})$ replete is equivalent to $\mathscr{F}$ replete. Moreover in such spaces, $\sigma(\mathscr{F})$ separates $s(\mathscr{F})$ so we can make further implications pertaining to $s(\mathscr{F})$-replete and $\sigma(\mathscr{F})$-replete.

Further applications in this manner are easy to give. We have just presented a few in order to give the flavor of one type of application that the general theorems are useful in handling.

5. Mappings. In this section we will present several general mapping theorems between spaces of regular lattice measures and then will show here and in the next section, how these results can be applied in a systematic fashion to a large number of topological mapping questions pertaining to preservation of various specific types of repleteness.

Throughout this section, $X$ and $Y$ will denote two abstract sets. $\mathscr{L}_{1}$ will denote a lattice of subsets of $X$ and $\mathscr{L}_{2}$ a lattice of subsets of $Y$.

Definition 5.1. A mapping $T: X \rightarrow Y$ is $\mathscr{L}_{1}-\mathscr{L}_{2}$ continuous if $T^{-1}\left(\mathscr{L}_{2}\right)$ is contained in $\mathscr{L}_{1}$. It is $\mathscr{L}_{1}-\mathscr{L}_{2}$ closed if $T\left(\mathscr{L}_{1}\right) \subset \mathscr{L}_{2}$ where $T\left(\mathscr{L}_{1}\right)=\left\{T\left(L_{1}\right): L_{1} \in \mathscr{L}_{1}\right\}$.

TheOREM 5.1. Let $T: X \rightarrow Y$ be $\mathscr{L}_{1}-\mathscr{L}_{2}$ continuous and let $T$ be a surjection. If $T^{-1}\left(\mathscr{L}_{2}\right)$ semiseparates $\mathscr{L}_{1}$ and if $\mathscr{L}_{1}$ and $\mathscr{L}_{2}$ are both delta normal lattices, then,

(a) The mapping $T^{*}: M R\left(\mathscr{L}_{1}\right) \rightarrow M R\left(\mathscr{L}_{2}\right)$ given by $T^{*} \mu=\mu T^{-1}$ is a surjection and is a bijection if $T^{-1}\left(\mathscr{L}_{2}\right)$ separates $\mathscr{L}_{1}$.

(b) If $T^{* *}$ is the restriction of $T^{*}$ to $M R\left(\sigma, \mathscr{L}_{1}\right)$ and if $\mathscr{L}_{1}$ is $T^{-1}\left(\mathscr{L}_{2}\right)$ countably paracompact or $\mathscr{L}_{1}$ is countably compact, then $T^{* *} \operatorname{maps} M R\left(\sigma, \mathscr{L}_{1}\right)$ onto $M R\left(\sigma, \mathscr{L}_{2}\right)$.

(c) $T^{*}$ is a continuous map with respect to the vague topologies.

Proof. (a) Using Theorem 4.1 (a) and the surjectivity of $T$ 
we see that $T^{*}$ is a mapping of $M R\left(\mathscr{L}_{1}\right)$ into $M R\left(\mathscr{L}_{2}\right)$. Suppose $\nu \in M R\left(\mathscr{L}_{2}\right)$. Define $\nu^{*}$ on $\mathscr{A}\left(T^{-1}\left(\mathscr{L}_{2}\right)\right)$ by $\nu^{*}\left(T^{-1}(B)\right)=\nu(B)$ where $B \in \mathscr{A}\left(\mathscr{L}_{2}\right) . \quad \nu^{*} \in M R\left(T^{-1}\left(\mathscr{L}_{2}\right)\right)$. By Theorem $4.2, \nu^{*}$ extends uniquely to a $\mu \in M R\left(\mathscr{L}_{1}\right)$. Clearly $\nu=\mu T^{-1}$ on $\mathscr{A}\left(\mathscr{L}_{2}\right)$, and we are done.

(b) follows immediately from (a) and Theorem 4.3.

(c) Suppose

$$
\begin{aligned}
& V\left(\nu_{0}, g_{1}, \cdots, g_{n}, \varepsilon\right) \\
& \quad=\left\{\nu \in M R\left(\mathscr{L}_{2}\right):\left|\int g_{i} d \nu-\int g_{i} d \nu_{0}\right|<\varepsilon, i=1,2, \cdots, n\right\}
\end{aligned}
$$

is a basic open set in $M R\left(\mathscr{L}_{2}\right)$ with the vague topology. Suppose $T\left(\mu_{0}\right)=\nu_{0}$. Then $f_{i}=g_{i} T \in C_{b}\left(\mathscr{L}_{1}\right)$ and $\int g_{i} d \nu=\int f_{i} d \mu$ for each $i=1,2, \cdots, n$, and any $\nu \in M R\left(\mathscr{L}_{2}\right)$ such that $T(\mu)=\nu$. It follows that the basic open set

$$
\begin{aligned}
& V\left(\mu_{0}, f_{1}, \cdots, f_{n}, \varepsilon\right) \\
& \quad=\left\{\mu \in M R\left(\mathscr{L}_{1}\right):\left|\int f_{i} d \mu-\int f_{i} d \mu_{0}\right|<\varepsilon, i=1,2, \cdots, n\right\}
\end{aligned}
$$

is mapped into $V\left(\nu_{0}, g_{1}, \cdots, g_{n}, \varepsilon\right)$ and thus $T$ is continuous.

CoRollary 5.1. Let the surjection $T: X \rightarrow Y$ be $\mathscr{L}_{1}-\mathscr{L}_{2}$ continuous and $\mathscr{L}_{1}-\mathscr{L}_{2}$ closed where $\mathscr{L}_{1}$ and $\mathscr{L}_{2}$ are delta normal lattices, then the corresponding conclusions of Theorem 5.1 remain valid.

Proof. It is easy to see in this case that $T^{-1}\left(\mathscr{L}_{2}\right)$ semiseparates $\mathscr{L}_{1}$.

REMARK 5.1. When $T^{*}$ of Theorem 5.1 is restricted to $\operatorname{IR}\left(\mathscr{L}_{1}\right)$ a simple direct argument shows that $T^{*}$ is continuous with respect to the Wallman topologies. In this case the delta normality of $\mathscr{L}_{1}$ and $\mathscr{L}_{2}$ are not necessary. Furthermore the extension arguments are achieved through the more elementary use of filters than through representation theorems. Thus we have the following:

THEOREM 5.2. Let $T: X \rightarrow Y$ be a surjection which is $\mathscr{L}_{1}-\mathscr{L}_{2}$ continuous and $\mathscr{L}_{1}-\mathscr{L}_{2}$ closed. Then

(a) The map $\hat{T}: \operatorname{IR}\left(\mathscr{L}_{1}\right) \rightarrow \operatorname{IR}\left(\mathscr{L}_{2}\right)$ given by $\hat{T}(\mu)=\mu T^{-1}$ is a surjection continuous with respect to the Wallman topologies.

(b) The mapping $\hat{T}^{*}: \operatorname{IR}\left(\sigma, \mathscr{L}_{1}\right) \rightarrow \operatorname{IR}\left(\sigma, \mathscr{L}_{2}\right)$ given by $\hat{T}^{*}(\mu)=$ $\mu T^{-1}$ is a surjection continuous with respect to the Wallman topologies if $\mathscr{L}_{1}$ is $T^{-1}\left(\mathscr{L}_{2}\right)$ countably paracompact or if $\mathscr{L}_{1}$ is countably compact. 
(c) The mappings in (a) and (b) are bijections if $T^{-1}\left(\mathscr{L}_{2}\right)$ separates $\mathscr{L}_{1}$.

Another general mapping theorem of considerable interest is the following.

THeOREM 5.3. Let $T: X \rightarrow Y$ be $\mathscr{L}_{1}-\mathscr{L}_{2}$ continuous.

(a) If $\mathscr{L}_{2}$ is a delta lattice and if $\sigma\left(\mathscr{L}_{2}\right)=\rho\left(\mathscr{L}_{2}\right)$ or $\sigma\left(\mathscr{L}_{2}\right) \subset$ $s\left(\mathscr{L}_{2}\right)$, or,

(b) If $T$ is a bijection and $\mathscr{L}_{2}$ semiseparates $T\left(\mathscr{L}_{1}\right)$; then $\mu \in M R\left(\sigma, \mathscr{L}_{1}\right)$ implies $\mu T^{-1} \in M R\left(\sigma, \mathscr{L}_{2}\right)$ and the map $T^{* *}$ taking $\mu$ into $\mu T^{-1}$ is continuous with respect to the vague topologies.

Proof. The continuity in each case follows as in Theorem 5.1. That $\mu T^{-1} \in M R\left(\sigma, \mathscr{L}_{2}\right)$ in part (a) follows immediately from Theorem 2.3. To show the same in (b) define $\nu_{0}(T(A))=\mu(A)$ for $T(A) \in$ $\mathscr{A}\left(T\left(\mathscr{L}_{1}\right)\right)$. Then $\nu_{0}=\mu T^{-1}$ on $\mathscr{A}\left(T\left(\mathscr{L}_{1}\right)\right)$ and $\nu_{0} \in M R\left(\sigma, T\left(\mathscr{L}_{1}\right)\right)$. If $\mathscr{L}_{2}$ semiseparates $T\left(\mathscr{L}_{1}\right)$ then $\mu T^{-1}$ restricted to $\mathscr{A}\left(\mathscr{L}_{2}\right)$ is in $M R\left(\sigma, \mathscr{L}_{2}\right)$ by Theorem 4.1.

In what follows we present a very significant general mapping theorem and some of its many corollaries. The general theorems which follow from it are extremely useful in a variety of contexts and have far reaching consequences. We need the following definitions.

Definition 5.2. If $A$ is a subset of $X$ and $\mathscr{L}$ is a lattice of subsets of $X$, then $A$ is called relatively $\mathscr{L}$-replete if $A$ is $\mathscr{L} \cap A$ replete where $\mathscr{L} \cap A=\{L \cap A: L \in \mathscr{L}\} . \quad A$ is called $\mathscr{L}$-compact (or just compact if the lattice is clear) if the lattice $\mathscr{L} \cap A$ is compact.

Definition 5.3. Let $T: X \rightarrow Y$ be a surjection which is $\mathscr{L}_{1}-\mathscr{L}_{2}$ continuous and $\mathscr{L}_{1}-\mathscr{L}_{2}$ closed and such that $T^{-1}\{y\}$ is $\mathscr{L}_{1}$-compact for any $y \in Y$. Then under these conditions $T$ is called $\mathscr{L}_{1}-\mathscr{L}_{2}$ perfect or simply perfect if the lattices involved are clear.

LEMma 5.1. If $T: X \rightarrow Y$ has the property that $T^{-1}\{y\}$ is relatively $\mathscr{L}_{1}$ replete for each $y \in Y$ where $\mathscr{L}_{1}$ is a delta lattice then if $\mu \in \operatorname{IR}\left(\sigma, \mathscr{L}_{1}\right)$ and if $\mathscr{H}=\left\{A \in \mathscr{L}_{1}: \mu(A)=1\right\}, \quad T\left(\cap A_{\alpha}\right)=\bigcap T\left(A_{\alpha}\right)$ where the $A_{\alpha}$ run through $\mathscr{H}$.

Proof. We need only show that $\bigcap T\left(A_{\alpha}\right) \subset T\left(\cap A_{\alpha}\right)$ where $A_{\alpha} \in \mathscr{L}$. Suppose $y \in \bigcap T\left(A_{\alpha}\right)$; then $T^{-1}\{y\} \cap A_{\alpha} \neq \varnothing$ for each $A_{\alpha} \in \mathscr{H}$. Indeed, if not then $A_{\alpha} \in\left(T^{-1}\{y\}\right)^{\prime}=T^{-1}\left(\{y\}^{\prime}\right)$ for each $A_{\alpha} \in \mathscr{H}$ and 
thus applying $T$ to both sides, $T\left(A_{\alpha}\right) \subset\{y\}^{\prime}$ for each $A_{\alpha} \in \mathscr{H}$ contradicting $y \in T\left(A_{\alpha}\right)$ for each $A_{\alpha} \in \mathscr{H}$. Thus the collection

$$
\mathscr{B}=\left\{T^{-1}\{y\} \cap A_{\alpha}: A_{\alpha} \in \mathscr{H}\right\}
$$

is an $\mathscr{L}_{1} \cap T^{-1}\{y\}$ filter with the countable intersection property. Moreover $\mathscr{B}$ is maximal since if $\left(T^{-1}\{y\} \cap C\right) \cap\left(T^{-1}\{y\} \cap A_{\alpha}\right) \neq \varnothing$ for each $A_{\alpha} \in \mathscr{H}$, where $C \in \mathscr{L}_{1}$; then $C \cap A_{\alpha} \neq \varnothing$ for each $A_{\alpha} \in \mathscr{H}$, and since $\mathscr{H}$ is maximal $C \in \mathscr{H}$. It follows that $T^{-1}\{y\} \cap C \in \mathscr{B}$ and thus $\mathscr{B}$ is a $T^{-1}\{y\} \cap \mathscr{L}_{1}$ ultrafilter with the countable intersection property. Since $T^{-1}\{y\}$ is relatively $\mathscr{L}_{1}$-replete $\mathscr{B}$ has nonempty intersection. If $x$ is in this intersection then $T(x)=y$ and the proof is complete since $x \in \bigcap A_{\alpha}$ where $\left\{A_{\alpha}\right\}$ runs through $\mathscr{H}$.

REMarK 5.2. By using Theorem 2.1 in conjunction with Lemma 5.1 one easily gets as corollary the following useful lemma of Meyer [41, p. 33, Theorem 6].

Lemma 5.2. If $T: X \rightarrow Y$ is such that $T^{-1}\{y\}$ is $T^{-1}\{y\} \cap \mathscr{L}_{1^{-}}$ countably compact for each $y \in Y$ then if $A_{n} \in \mathscr{L}_{1}$ and $A_{n} \downarrow \varnothing$, $T\left(A_{n}\right) \downarrow \varnothing$.

THEOREM 5.4. Let $T: X \rightarrow Y$ be $\mathscr{L}_{1}-\mathscr{L}_{2}$ continuous and $\mathscr{L}_{1}-\tau \sigma\left(\mathscr{L}_{2}\right)$ closed where $\mathscr{L}_{1}$ is a delta lattice and $\mathscr{L}_{2}$ is a separating disjunctive delta lattice with $\sigma\left(\mathscr{L}_{2}\right) \subset s\left(\mathscr{L}_{2}\right)$. Let $\mathscr{L}_{3} \supset \mathscr{L}_{1}$ be a lattice of subsets of $X$ such that $\mathscr{L}_{1} \subset \mathscr{L}_{3} \subset \tau\left(\mathscr{L}_{1}\right)$ and such that any $\mu \in I R\left(\sigma, \mathscr{L}_{3}\right)$ when restricted to $\mathscr{A}\left(\mathscr{L}_{1}\right)$ is in $\operatorname{IR}\left(\sigma, \mathscr{L}_{1}\right)$ (e.g., if $\sigma\left(\mathscr{L}_{1}\right) \subset s\left(\mathscr{L}_{1}\right)$ or if $\mathscr{L}_{1}$ semiseparates $\left.\mathscr{L}_{3}\right)$. Then if $T^{-1}\{y\}$ is relatively $\mathscr{L}_{1}$ replete for each $y \in Y, \mathscr{L}_{2}$ replete implies $\mathscr{L}_{3}$ replete.

Proof. Suppose $\mu \in \operatorname{IR}\left(\sigma, \mathscr{L}_{3}\right)$; by hypothesis, the restriction $\mu_{1}$ of $\mu$ to $\mathscr{A}\left(\mathscr{L}_{1}\right)$ is in $\operatorname{IR}\left(\sigma, \mathscr{L}_{1}\right)$. We may assume that $\mu_{1}$ is defined on $\sigma\left(\mathscr{L}_{1}\right)$. Define $\nu_{1}=\mu_{1} T^{-1}$ on $\mathscr{A}\left(\mathscr{L}_{2}\right) . \quad \nu_{1} \in I R\left(\sigma, \mathscr{L}_{2}\right)$ since $\sigma\left(\mathscr{L}_{2}\right) \subset$ $s\left(\mathscr{L}_{2}\right)$ and $\nu_{1}$ is fixed at a single point $p$. Its unique $\mathscr{L}_{2}$-regular extension to $\sigma\left(\mathscr{L}_{2}\right)$ also denoted by $\nu_{1}$ is also concentrated at $p$. Let $\mathscr{H}_{1}=\left\{A \in \mathscr{L}_{1}: \mu_{1}(A)=1\right\}$. Since $T(A) \in \tau \sigma\left(\mathscr{L}_{2}\right)$ for each $A \in \mathscr{L}_{1}$ we may write for any $A \in \mathscr{H}_{1}, T(A)=\bigcap C_{\alpha}$ where $C_{\alpha} \in \sigma\left(\mathscr{L}_{2}\right)$. For any such $C_{\alpha}$ we have $\nu_{1}\left(C_{\alpha}\right)=\mu_{1} T^{-1}\left(C_{\alpha}\right) \geqq \mu_{1}\left(A_{1}\right)=1$. Thus $p \in C_{\alpha}$ for each such $C_{\alpha}$; hence $p \in T(A)$ for any $A \in \mathscr{H}_{1}$. By Lemma $5.1 \mu_{1}$ is fixed and since $\mathscr{L}_{3} \subset \tau\left(\mathscr{L}_{1}\right)$ clearly $\mu$ is fixed. Thus $\mathscr{L}_{3}$ is replete.

Taking $\mathscr{L}_{1}=\mathscr{L}_{3}$ in Theorem 5.4 we get

Theorem 5.5. Let $T: X \rightarrow Y$ be $\mathscr{L}_{1}-\mathscr{L}_{2}$ continuous and $\mathscr{L}_{1}-\tau\left(\sigma\left(\mathscr{L}_{2}\right)\right)$ closed where $\mathscr{L}_{1}$ is a delta lattice and $\mathscr{L}_{2}$ is a separating disjunctive delta lattice with $\sigma\left(\mathscr{L}_{2}\right) \subset s\left(\mathscr{L}_{2}\right)$. Then if 
$T^{-1}\{y\}$ is relatively $\mathscr{L}_{1}$ replete for each $y \in Y, \mathscr{L}_{2}$ replete implies $\mathscr{L}_{1}$-replete. (In particular if $T^{-1}\{y\}$ is compact for each $y \in Y$, then $\mathscr{L}_{2}$ replete implies $\mathscr{L}_{1}$ replete.) We note that in the case $T^{-1}\{y\}$ is compact for each $y \in Y$ one may relax the condition that $\mathscr{L}_{1}$ be a delta lattice since $\cap \mathscr{B}$ in Lemma 5.1 is nonempty.

Let us specialize to the case where $E \subset X$ and $T: E \rightarrow X$ is the identity map. If $\mathscr{L}$ is a separating disjunctive delta lattice of subsets of $X$ with $\sigma(\mathscr{L}) \subset s(\mathscr{L})$ and $\mathscr{L}_{E}$ is a lattice of subsets of $E$ with $\mathscr{L} \cap E \subset \mathscr{L}_{E}$, then obviously $T$ is $\mathscr{L}_{E}-\mathscr{L}$ continuous. If in addition $\mathscr{L}_{E} \subset \tau \sigma(\mathscr{L})$ then $T$ is also $\mathscr{L}_{E}-\tau \sigma(\mathscr{L})$ closed. Since $T$ is an injection $T^{-1}\{y\}$ is clearly relatively replete and the conditions of Theorem 5.5 hold. Thus we have

THEOREM 5.6. If $E \subset X$ and $\mathscr{L}$ is a separating disjunctive delta lattice of subsets of $X$ with $\sigma(\mathscr{L}) \subset s(\mathscr{L})$ and if $\mathscr{L}_{E}$ is a lattice of subsets of $E$ with $\mathscr{L} \cap E \subset \mathscr{L}_{E}$ then if $\mathscr{L}_{E} \subset \tau \sigma(\mathscr{L})$, $\mathscr{L}$ replete implies $\mathscr{L}_{E}$ replete. (In particular if $E \in \tau \sigma(\mathscr{L})$ and $\mathscr{L}_{E}=$ $\mathscr{L} \cap E$, then $\mathscr{L}$ replete implies $\mathscr{L} \cap E$ replete.)

REMARK 5.3. If $T: X \rightarrow Y$ is $\mathscr{L}_{1}-\mathscr{L}_{2}$ continuous, $\mathscr{L}_{1}-\mathscr{L}_{2}$ closed and onto, then we may relax the condition $\sigma\left(\mathscr{L}_{2}\right) \subset s\left(\mathscr{L}_{2}\right)$ in Theorem 5.4 since the measure $\nu_{1}$ constructed in the proof of Theorem 5.4 is necessarily $\mathscr{L}_{2}$ regular. Thus we have

Theorem 5.7. If $T: X \rightarrow Y$ is $\mathscr{L}_{1}-\mathscr{L}_{2}$ continuous, $\mathscr{L}_{1}-\tau \sigma\left(\mathscr{L}_{2}\right)$ closed and onto where $\mathscr{L}_{1}$ is a delta lattice and $\mathscr{L}_{2}$ is a separating disjunctive delta lattice then if $T^{-1}\{y\}$ is relatively $\mathscr{L}_{1}$-replete for each $y \in Y, \mathscr{L}_{2}$ replete implies $\mathscr{L}_{1}$-replete. (In particular if $T$ is $\mathscr{L}_{1}-\mathscr{L}_{2}$ perfect $\mathscr{L}_{2}$-replete implies $\mathscr{L}_{1}$ replete.)

Some further corollaries follow.

CoROLlary 5.2. If $X, Y$ are Tychonoff spaces and if $T: X \rightarrow Y$ is a continuous $Z$-map (i.e. $\left.T\left(\mathscr{Z}_{1}\right) \subset \mathscr{F}_{2}\right)$, with the property that $T^{-1}\{y\}$ is realcompact and a $Z$-embedded subset of $X$ for each $y \in Y$, (i.e., every zero set of $T^{-1}\{y\}$ is the trace of a zero set of $X$ ), then, if $Y$ is realcompact then $X$ is realcompact.

Proof. In Theorem 5.4 let $\mathscr{L}_{1}=\mathscr{L}_{3}=\mathscr{Z}_{1}, \mathscr{L}_{2}=\mathscr{Z}_{2} . \quad T$ is clearly $\mathscr{L}_{1}-\mathscr{L}_{2}$ continuous and $\mathscr{L}_{1}-\tau\left(\mathscr{L}_{2}\right)=\mathscr{F}_{2}$ closed. Finally $T^{-1}\{y\}$ is relatively $\mathscr{L}_{1}$-replete because it is realcompact and $Z$-embedded.

REMARK 5.4. Corollary 5.2 strengthens Theorem 5.3 in Isiwata [31]. 
CoRollary 5.3 (Varadarajan-Moran [51], [42]). If $X$ is a realcompact Tychonoff space and $E \subset X$ is in $\tau \sigma(\mathscr{Z})$ then $E$ is realcompact. (In particular a Baire subset of a realcompact space is realcompact.)

Proof. In Theorem 5.6 take $\mathscr{L}_{E}$ to be the zero sets of $E$ and $\mathscr{L}=\mathscr{Z}$, the zero sets of $X$. To see that $\mathscr{L}_{E} \subset \tau \sigma(\mathscr{L})$ take a $Z \in \mathscr{L}_{E}$;

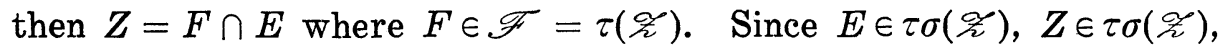
hence $\mathscr{L}_{E} \subset \tau \sigma(\mathscr{\varkappa})$.

REMARK 5.5. If $X-\{x\} \in \sigma(\mathscr{\varkappa})$ for each $x \in X$ for $X$ a realcompact Tychonoff space, then every subset $E$ of $X$ is in $\tau \sigma(\mathscr{Z})$ and thus by Corollary 5.3 every subset is realcompact.

CoRollary 5.4 (Hager-Reynolds-Rice [24]). If $T: X \rightarrow Y$ is a Borel measurable injection then if $Y$ is Borel replete, $X$ is Borel replete. (In particular by taking $X \subset Y$ and $T$ to be the identity map we get that any subset of a Borel replete space is Borel replete.)

Proof. In Theorem 5.5 take $\mathscr{L}_{1}=\sigma\left(\mathscr{F}_{1}\right), \mathscr{L}_{2}=\sigma\left(\mathscr{F}_{2}\right)$. Note that $T\left(\mathscr{L}_{1}\right)$ is always contained in $\tau \sigma\left(\mathscr{F}_{2}\right)=$ all subsets of $Y$, regardless of what $\mathscr{L}_{1}$ is.

Corollary 5.5 (Dykes [16]). If $X, Y$ are Tychonoff spaces and $T: X \rightarrow Y$ is perfect then if $Y$ is $\mathscr{F}_{2}$ replete then $X$ is $\mathscr{F}_{1}$ replete.

(The converse follows simply from Theorem 5.2 together with Lemma 5.2.)

Proof. In Theorem 5.7 take $\mathscr{L}_{1}=\mathscr{F}_{1}, \mathscr{L}_{2}=\mathscr{F}_{2}$.

Corollary 5.6. If a c.b. space $E$ is contained in a Borel replete Tychonoff space $X$ then $E$ is realcompact.

Proof. By a previous Corollary, $E$ is Borel replete and thus by Theorem 4.2 F -replete. The corollary now follows from the remarks after (4.4).

One can get many more subspace and mapping theorems concerning repleteness as special cases of Theorems 5.4-5.7. By combining these theorems with our general extension theorems, still more general theorems may be obtained. We do not pursue this however, since the general techniques and assumptions necessary should be clear. We will just consider one specialized case of particular interest and note some of its significant consequences. 
THeOREM 5.8. Let $\mathscr{L}_{1}$ and $\mathscr{L}_{3}$ be lattices of subsets of $X$ such that $\mathscr{L}_{1} \subset \mathscr{L}_{3} \subset \tau\left(\mathscr{L}_{1}\right)$ and $\sigma\left(\mathscr{L}_{1}\right) \subset s\left(\mathscr{L}_{1}\right)$. Let $\mathscr{L}_{2} \subset \mathscr{L}_{4}$ be lattices of subsets of $Y$ such that $\mathscr{L}_{4}$ is $\mathscr{L}_{2}$ countably paracompact or a c.b $\mathscr{L}_{2}$ lattice. Let $T$ be an $\mathscr{L}_{3}-\mathscr{L}_{4}$ continuous closed surjection. If $\mathscr{L}_{3}$ is $T^{-1}\left(\mathscr{L}_{4}\right)$ countably paracompact, then $\mathscr{L}_{1}$-replete implies $\mathscr{L}_{2}$ replete.

Proof. Since $\mathscr{L}_{1}$ is replete and $\mathscr{L}_{1} \subset \mathscr{L}_{3} \subset \tau\left(\mathscr{L}_{1}\right)$ we have that $\mathscr{L}_{3}$ is replete. Now let $\nu \in I R\left(\sigma, \mathscr{L}_{2}\right)$. $\nu$ can be extended to a $\rho \in \operatorname{IR}\left(\sigma, \mathscr{L}_{4}\right)$ by Theorem 3.4 (a). Also $\rho=\mu T^{-1}$ where $\mu \in \operatorname{IR}\left(\sigma, \mathscr{L}_{3}\right)$ by Theorem 5.2 (b). Since $\mu$ is fixed, we have, as is easily seen, that $\rho$ is fixed.

Before giving some consequences of this, we note:

Lemma 5.3. If $T: X \rightarrow Y$ is $\mathscr{L}_{1}-\mathscr{L}_{2}$ perfect and if $\mathscr{L}_{2}$ is countably paracompact, then $\mathscr{L}_{1}$ is $T^{-1}\left(\mathscr{L}_{2}\right)$ countably paracompact.

Proof. If $F_{n} \downarrow \varnothing$ where $F_{n} \in \mathscr{L}_{1}$ then by Lemma $5.2 T\left(F_{n}\right) \downarrow \varnothing$. Thus there are $G_{n}^{\prime}, G_{n} \in \mathscr{L}_{2}$ with $T\left(F_{n}\right) \subset G_{n}^{\prime}$ and $G_{n}^{\prime} \downarrow \varnothing$. We therefore have $F_{n} \subset\left(T^{-1}\left(G_{n}\right)\right)^{\prime} \downarrow \varnothing$.

CoRollary 5.7. If $X$ and $Y$ are Tychonoff spaces and $T: X \rightarrow Y$ is a perfect map, then if $Y$ is countably paracompact and normal or a c.b space then $X$ realcompact implies $Y$ realcompact.

Proof. In the theorem take $\mathscr{L}_{3}=\mathscr{F}_{1}, \mathscr{L}_{4}=\mathscr{F}_{2}, \mathscr{L}_{1}=\mathscr{Z}_{1}$, $\mathscr{L}_{2}=\mathscr{Z}_{2}$.

CoRollary 5.8 (Frolik-Isiwata). Let $X$ and $Y$ be Tychonoff spaces and let $T: X \rightarrow Y$ be perfect. If $X$ is countably paracompact and normal or a c.b space, then $X$ realcompact implies $Y$ realcompact.

Proof. This follows from the previous corollary observing that countably paracompact and normal is preserved under continuous closed surjections.

Corollary 5.9 (Frolik). Realcompactness is preserved under an open perfect mapping.

Proof. If $T: X \rightarrow Y$ is perfect where $X$ and $Y$ are Tychonoff spaces then of course $T^{-1}\left(\mathscr{Z}_{2}\right) \subset \mathscr{Z}_{1}$ and $T^{-1}\{y\}$ is $\mathscr{Z}_{1}$-compact for each $y \in Y$. Also since $T$ is open it is not difficult to see that $T\left(\mathscr{Z}_{1}\right) \subset \mathscr{Z}_{2}$. 
To complete the proof we need only take in the theorem $\mathscr{L}_{1}=\mathscr{L}_{3}=\mathscr{Z}_{1}$, $\mathscr{L}_{2}=\mathscr{L}_{4}=\mathscr{Z}_{2}$.

REMARK 5.6. For completeness we will consider here the relationship of some of the previous work with almost realcompactness. Returning to the abstract framework, let $T: X \rightarrow Y$ be $\mathscr{L}_{1}-\mathscr{L}_{2}$ perfect. Let $\nu \in I R\left(\mathscr{L}_{2}^{\prime}\right) \cap I\left(\sigma^{*}, \mathscr{L}_{2}\right)$. Then there exists a $\mu \in I R\left(\mathscr{L}_{1}^{\prime}\right)$ such that $\mu T^{-1}(A)=\nu(A)$ where $A \in \mathscr{A}\left(\mathscr{L}_{2}^{\prime}\right)=\mathscr{A}\left(\mathscr{L}_{2}\right)$. Thus $\mu T^{-1}=\nu$ on $\mathscr{L}_{2}$. By Lemma 5.2 if $\nu \in I\left(\sigma^{*}, \mathscr{L}_{2}\right)$ then $\mu \in I\left(\sigma^{*}, \mathscr{L}_{1}\right)$. Thus if $\mu$ is fixed, $\nu$ is fixed.

Conversely let $\mu \in I R\left(\mathscr{L}_{1}^{\prime}\right) \cap I\left(\sigma^{*}, \mathscr{L}_{1}\right)$ and define $\nu=\mu T^{-1}$ on $\mathscr{A}\left(\mathscr{L}_{2}\right)$. Then $\nu \in I\left(\sigma^{*}, \mathscr{L}_{2}\right)$. If $\mathscr{L}_{2}$ is regular then by $3.5, \nu$ is fixed and by the perfectness and Lemma $5.2 \mu$ is fixed. Thus we have:

TheOREM 5.8. Let $T: X \rightarrow Y$ be $\mathscr{L}_{1}-\mathscr{L}_{2}$ perfect.

(a) If $X$ is $\mathscr{L}_{1}$-almost replete then $Y$ is $\mathscr{L}_{2}$ almost replete.

(b) If $Y$ is $\mathscr{L}_{2}$ almost replete and $\mathscr{L}_{2}$ is regular, then $X$ is $\mathscr{L}_{1}$-almost replete.

As a corollary of this we get of course Frolik's result that for regular spaces almost realcompactness is preserved under perfect mappings.

We note that further generalizations of some of the corollaries given have been obtained (see e.g., [16], [31], [11]). We could indeed generalize slightly some of our abstract results in line with those but most of this brings us into the realm of $\tau$-smooth measures and will not be considered here.

6. Further mapping theorems. We turn to some further general abstract mapping theorems which have a great number of application.

We noted earlier in Theorem 5.3, general conditions under which $T: X \rightarrow Y$ induces a continuous map $T^{* *}: M R\left(\sigma, \mathscr{L}_{1}\right) \rightarrow M R\left(\sigma, \mathscr{L}_{2}\right)$. If we specialize part of this theorem to the case of zero-one measures and use a remark analogous to Remark 5.1 then we have

Theorem 6.1. If $T: X \rightarrow Y$ is $\mathscr{L}_{1}-\mathscr{L}_{2}$ countinuous, and if $\mathscr{L}_{2}$ is a delta lattice such that $(*) \sigma\left(\mathscr{L}_{2}\right) \subset s\left(\mathscr{L}_{2}\right)$ then the map $T_{1}: \operatorname{IR}\left(\sigma, \mathscr{L}_{1}\right) \rightarrow \operatorname{IR}\left(\sigma, \mathscr{L}_{2}\right)$ given by $T_{1}(\mu)=\mu T^{-1}$ is continuous with respect to the Wallman topologies.

Let us specialize still further. We assume that both $\mathscr{L}_{1}$ and $\mathscr{L}_{2}$ are separating disjunctive delta lattices. In this case, $X$ and $Y$ with the respective topologies $\tau\left(\mathscr{L}_{1}\right)$ and $\tau\left(\mathscr{L}_{2}\right)$ are densely embedded in 
their respective repletions $I R\left(\sigma, \mathscr{L}_{1}\right)$ and $\operatorname{IR}\left(\sigma, \mathscr{L}_{2}\right)$. Now let $D \subset Y$ and let $E=T^{-1}(D)$. Let $\mathscr{L}_{D}$ and $\mathscr{L}_{E}$ be basic separating disjunctive delta lattices of subsets of $D$ and $E$ respectively. Consider the inclusion map $i: E \subset X$, and suppose that $\mathscr{L}_{1} \cap E \subset \mathscr{L}_{E}$ so that $i$ is $\mathscr{L}_{E}-\mathscr{L}_{1}$ continuous. Clearly $\mathscr{L}_{1} \cap E$ satisfies (*) of Theorem 6.1 if $\mathscr{L}_{1}$ does. Consider the map $i_{1}: \operatorname{IR}\left(\sigma, \mathscr{L}_{E}\right) \rightarrow \operatorname{IR}\left(\sigma, \mathscr{L}_{1}\right)$ of Theorem 6.1. Then if $X$ is $\mathscr{L}_{1}$ replete, $i_{1}: \operatorname{IR}\left(\sigma, \mathscr{L}_{E}\right) \rightarrow X$. Next the restriction $T^{\prime}$ of $T$ to $E$ maps $E$ into $D$ and $T^{\prime}=T \circ i$. If $T^{-1}\left(\mathscr{L}_{D}\right) \subset \mathscr{L}_{E}$ and $\mathscr{L}_{D}$ satisfies (*) then $T_{1}^{\prime}: \operatorname{IR}\left(\sigma, \mathscr{L}_{E}\right) \rightarrow \operatorname{IR}\left(\sigma, \mathscr{L}_{D}\right)=D$ if $D$ is $\mathscr{L}_{D}$-replete. By denseness $T_{1}^{\prime}=(T \circ i)_{1}=T \circ i_{1}$ and by a standard elementary denseness and continuity result ([22], p. 92) $i_{1}\left(I R\left(\sigma, \mathscr{L}_{E}\right)-E\right) \subset X-E$ and thus $T_{1}^{\prime}\left(I R\left(\sigma, \mathscr{L}_{E}\right)-E\right) \subset T(X-E) \subset Y-D$. Also

$$
T_{1}^{\prime}\left(I R\left(\sigma, \mathscr{L}_{E}\right)-E\right) \subset D .
$$

Therefore $\operatorname{IR}\left(\sigma, \mathscr{L}_{E}\right)=E$. Summarizing we get:

TheOREM 6.2. Let $T: X \rightarrow Y$ be $\mathscr{L}_{1}-\mathscr{L}_{2}$ continuous where $\mathscr{L}_{1}$ and $\mathscr{L}_{2}$ are basic separating disjunctive delta lattices such that $\sigma\left(\mathscr{L}_{1}\right) \subset s\left(\mathscr{L}_{1}\right)$ and $\sigma\left(\mathscr{L}_{2}\right) \subset s\left(\mathscr{L}_{2}\right)$. If $D \subset Y$ and $E=T^{-1}(D)$, and if $\mathscr{L}_{D}$ and $\mathscr{L}_{E}$ are basic separating disjunctive lattices of subsets of $D$ and $E$ respectively such that $\mathscr{L}_{1} \cap E \subset \mathscr{L}_{E}, T^{-1}\left(\mathscr{L}_{D}\right) \subset \mathscr{L}_{E}$ and $\sigma\left(\mathscr{L}_{D}\right) \subset s\left(\mathscr{L}_{D}\right)$, then if $X$ is $\mathscr{L}_{1}$-replete, and $D$ is $\mathscr{L}_{D}$-replete, it follows that $E$ is $\mathscr{L}_{E}$-replete.

REMARK 6.1. In general if $X$ is an abstract set and $\mathscr{L}$ is a lattice of subsets and if $B \in \sigma(\mathscr{L})$, then it is a routine set theoretic argument to show that $W(\sigma(\mathscr{L}))=\sigma W(\mathscr{L})$ where here

$$
W(B)=\{\mu \in \operatorname{IR}(\sigma, \mathscr{L}): \mu(B)=1\},
$$

and $W(\sigma(\mathscr{L}))$ and $\sigma(W(\mathscr{L}))$ have the obvious meaning.

Next if we follow the terminology and notations immediately after Definition 3.1, we have that if $\mathscr{L}$ is strongly normal delta lattice and if $f \in C(\mathscr{L})$ has $L$ as a zero set, i.e.,

$$
L=Z(f)=\{x \in X: f(x)=0\},
$$

then $W(L)=Z\left(f^{*}\right)$ where $f^{*}$ is the continuous extension of $f$ to $\operatorname{IR}(\sigma, \mathscr{L})$ (see [49]) and from this it follows that $\{W(L): L \in \mathscr{L}$, $L=Z(f)$ for $f \in C(\mathscr{L})\}$ gives the totality of zero sets of $\operatorname{IR}(\sigma, \mathscr{L})$.

Corollary 6.1 (Moran [42]). Let $X$ and $Y$ be Tychonoff spaces and let $T: X \rightarrow Y$ be Baire measurable (i.e., $T^{-1}\left(\sigma\left(\mathscr{L}_{2}\right)\right) \subset \sigma\left(\mathscr{L}_{1}\right)$ where $\mathscr{L}_{1}$ is the collection of zero sets of $X$ and $\mathscr{L}_{2}$ is the collection of zero sets of $Y$ ). Then 
(a) there exists a unique Baire measurable extension $T^{*}$ of $T$ from $\nu X$ to $\nu Y$;

(b) the inverse image under $T$ of every relatively realcompact (i.e., ( $\mathscr{Z}_{2} \cap D$-replete)) subset of $Y$ is realcompact if $X$ is realcompact.

Proof. (a) Follows directly from Theorem 6.1 in conjunction with Remark 6.1 while (b) follows directly from Theorem 6.2 by taking $\mathscr{L}_{1}=\sigma\left(\mathscr{\varkappa}_{1}\right), \mathscr{L}_{2}=\sigma\left(\mathscr{Z}_{2}\right), \mathscr{L}_{E}=\sigma\left(\mathscr{\varkappa}_{E}\right)$ where $\mathscr{\varkappa}_{E}$ is the collection of zero sets of $E$ and $\mathscr{L}_{D}=\sigma\left(\mathscr{\varkappa}_{2}\right) \cap D$.

CoRollary 6.2 (Gillman-Jerrison [22]). Let $X$ and $Y$ be Tychonoff spaces. If $T: X \rightarrow Y$ is continuous and if $X$ is realcompact, then the inverse image of any realcompact subset of $Y$ is realcompact.

Proof. This follows from (b) of the previous corollary or may be obtained directly from Theorem 6.2 by taking $\mathscr{L}_{1}=\mathscr{\varkappa}_{1}, \mathscr{L}_{2}=\mathscr{\varkappa}_{2}$, $\mathscr{L}_{D}=\mathscr{\varkappa}_{D}$ and $\mathscr{L}_{E}=\mathscr{\varkappa}_{E}$ where $\mathscr{L}_{1}, \mathscr{L}_{2}, \mathscr{\varkappa}_{D}$ and $\mathscr{\varkappa}_{E}$ are the zero sets respectively of $X, Y, D$, and $E$.

REMARK 6.2. In Theorem 6.1 we obtained conditions under which an $\mathscr{L}_{1}-\mathscr{L}_{2}$ continuous function could be extended to the respective repletions $\operatorname{IR}\left(\sigma, \mathscr{L}_{1}\right)$ and $\operatorname{IR}\left(\sigma, \mathscr{L}_{2}\right)$ of $X$ and $Y$. We consider here because of its importance (see e.g., [28]) the "extension" of an $\mathscr{L}_{1}-\mathscr{L}_{2}$ continuous $T: X \rightarrow Y$ to a $T^{*}: \operatorname{IR}\left(\mathscr{L}_{1}\right) \rightarrow \operatorname{IR}\left(\mathscr{L}_{2}\right)$. As in the proof of Lemma 3.1 , let $\mu \in I R\left(\mathscr{L}_{1}\right)$. Then $\mu$ restricted to $T^{-1}\left(\mathscr{L}_{2}\right)$ is in $\operatorname{IR}\left(T^{-1}\left(\mathscr{L}_{2}\right)\right)$ if $T^{-1}\left(\mathscr{L}_{2}\right)$ semiseparates $\mathscr{L}_{1}$. Define $\nu(A)=\mu T^{-1}(A)$ for $A \in \mathscr{A}\left(\mathscr{L}_{2}\right)$. If $T$ is a surjection then $\nu \in I R\left(\mathscr{L}_{2}\right)$. As in Theorem 5.1 only using the simpler extension process of Theorem 3.1 and Remark 5.1 we get that any $\nu \in I R\left(\mathscr{L}_{2}\right)$ is of this form. Therefore we have:

THeorem 6.3. If $T: X \rightarrow Y$ is an $\mathscr{L}_{1}-\mathscr{L}_{2}$ continuous surjection such that $T^{-1}\left(\mathscr{L}_{2}\right)$ semiseparates $\mathscr{L}_{1}$, then the map $T^{*}: \operatorname{IR}\left(\mathscr{L}_{1}\right) \rightarrow$ $\operatorname{IR}\left(\mathscr{L}_{2}\right)$ given by $T^{*} \mu=\mu T^{-1}$ is a surjection and is continuous with respect to the Wallman topologies. If $\mathscr{L}_{1}$ and $\mathscr{L}_{2}$ are separating disjunctive lattices which are bases for the topologies $\tau\left(\mathscr{L}_{1}\right)$ and $\tau\left(\mathscr{L}_{2}\right)$ then $X$ and $Y$ are embedded in $\operatorname{IR}\left(\mathscr{L}_{1}\right)$ and $\operatorname{IR}\left(\mathscr{L}_{2}\right)$ respectively and $T^{*}$ extends $T$.

In what follows the closure will be taken with respect to the topologies $\tau\left(\mathscr{L}_{1}\right)$ and $\tau\left(\mathscr{L}_{2}\right)$. Let $T: X \rightarrow Y$ be $\mathscr{L}_{1}-\mathscr{L}_{2}$ continuous and $\mu \in \operatorname{IR}\left(\mathscr{L}_{1}\right)$. Define $\mu T^{-1}$ on $\mathscr{A}\left(\mathscr{L}_{2}\right)$. Again we want conditions which will insure that $T$ induces a continuous map from $\operatorname{IR}\left(\mathscr{L}_{1}\right) \rightarrow$ 
$\operatorname{IR}\left(\mathscr{L}_{2}\right)$. Assume that $\mathrm{cl}\left(T\left(\mathscr{L}_{1}\right)\right) \subset \mathscr{L}_{2}$ where $\mathrm{cl}\left(T\left(L_{1}\right)\right)$ is the closure of $T\left(L_{1}\right)$ in $Y$ and $\operatorname{cl}\left(T\left(\mathscr{L}_{1}\right)\right)=\left\{\operatorname{cl}\left(T\left(L_{1}\right)\right): L_{1} \in \mathscr{L}_{1}\right\}$. This would certainly be the case for example if $T$ is $\mathscr{L}_{1}-\mathscr{L}_{2}$ closed.

Suppose also that $T$ satisfies condition $\left({ }^{* *}\right)$ : Whenever $B_{1}^{\prime} \cup B_{2}^{\prime} \cup$ $\cdots \cup B_{n}^{\prime}=Y$ where $B_{1}, B_{2}, \cdots, B_{n} \in \mathscr{L}_{2}$ there exists $A_{1}, A_{2}, \cdots, A_{m} \in \mathscr{L}_{1}$ such that $X=A_{1}^{\prime} \cup A_{2}^{\prime} \cup \cdots \cup A_{m}^{\prime}$ and for each $A_{k}^{\prime}, k=1,2,3, \cdots, m$, and any $C \subset A_{k}^{\prime}, C \in \mathscr{L}_{1}$ implies there exists a $B_{r}^{\prime}, r=1,2, \cdots, n$, such that $\mathrm{cl}(T(C)) \subset B_{r}^{\prime}$.

If $\mu \in I R\left(\mathscr{L}_{1}\right)$ then $\mu T^{-1} \in I\left(\mathscr{L}_{2}\right)$ and there exists a $\nu \in I R\left(\mathscr{L}_{2}\right)$ such that $\mu T^{-1} \leqq \nu$ on $\mathscr{L}_{2}$. We show first that under condition $\left({ }^{* *}\right) \nu$ is unique. (Note: uniqueness follows from (3.3) if $\mathscr{L}_{2}$ is normal.) Suppose $\rho \in I R\left(\mathscr{L}_{2}\right)$ and $\mu T^{-1} \leqq \rho$ on $\mathscr{L}_{2}$, and $\nu \neq \rho$. Then there exists $B_{1}, B_{2} \in \mathscr{L}_{2}$ such that $B_{1} \cap B_{2}=\varnothing, \nu\left(B_{1}\right)=1, \rho\left(B_{1}\right)=0$ and $\rho\left(B_{2}\right)=1$, $\nu\left(B_{2}\right)=0$. Thus, $B_{1}^{\prime} \cup B_{2}^{\prime}=Y$ and by $\left({ }^{* *}\right)$ there are $A_{1}, \cdots, A_{m} \in \mathscr{L}_{1}$, such that $X=A_{1}^{\prime} \cup A_{2}^{\prime} \cup \cdots \cup A_{m}^{\prime}$. Hence for some $k=1,2, \cdots, m$, $\mu\left(A_{k}^{\prime}\right)=1$. By regularity there is an $A \in \mathscr{L}_{1}, A \subset A_{k}^{\prime}$ and $\mu(A)=1$. By (**) either $\operatorname{cl}(T(A)) \subset B_{1}^{\prime}$ or $\operatorname{cl}(T(A)) \subset B_{2}^{\prime}$. If $\operatorname{cl}(T(A)) \subset B_{1}^{\prime}$, then $\nu(\mathrm{cl}(T(A))) \geqq \mu T^{-1}(\mathrm{cl}(T(A))) \geqq \mu(A)=1$, so $\nu\left(B_{1}^{\prime}\right)=1$, a contradiction. Similarly, cl $(T(A)) \subset B_{2}^{\prime}$ leads to a contradiction Thus $\nu$ is unique, and we have a well defined map $T_{*}: \operatorname{IR}\left(\mathscr{L}_{1}\right) \rightarrow \operatorname{IR}\left(\mathscr{L}_{2}\right)$ given by $\mu \rightarrow \nu$ where $\nu$ is the unique element of $\operatorname{IR}\left(\mathscr{L}_{2}\right)$ which is $\geqq \mu T^{-1}$ on $\mathscr{L}_{2}$.

Next we show that $T_{*}$ is continuous. Let $\nu \in I R\left(\mathscr{L}_{2}\right)$ and let $B \in \mathscr{L}_{2}$. If $\nu \in W(B)^{\prime}=\left\{\rho \in I R\left(\mathscr{L}_{2}\right): \rho(B)=0\right\}$ (a basic open set in $\left.\operatorname{IR}\left(\mathscr{L}_{2}\right)\right)$, then $\nu\left(B^{\prime}\right)=1$ and by regularity there is a $C \in \mathscr{L}_{2}$ such that $C \subset B^{\prime}$ and $\nu(C)=1$. Clearly $Y=C^{\prime} \cup B^{\prime}$ and as above there are $A, A_{k} \in \mathscr{L}_{1}$ with $A \subset A_{k}^{\prime}, \mu(A)=\mu\left(A_{k}^{\prime}\right)=1$, where $\operatorname{cl}(T(A)) \subset C^{\prime}$ or $\operatorname{cl}(T(A)) \subset B^{\prime}$. If $\operatorname{cl}(T(A)) \subset C^{\prime}$ then $\nu\left(\operatorname{cl}(T(A))=1\right.$ and $\nu\left(C^{\prime}\right)=1$, a contradiction. Thus $\operatorname{cl}(T(A)) \subset B^{\prime} . \quad \mu \in W\left(A_{k}^{\prime}\right)$. If $\lambda \in W\left(A_{k}^{\prime}\right)$ then $\lambda\left(A^{*}\right)=1$ for some $A^{*} \subset A_{k}^{\prime}$ where $A^{*} \in \mathscr{L}$. Thus $\left(\operatorname{cl}\left(T\left(A^{*}\right)\right) \subset B^{\prime}\right.$ and if $T_{*} \lambda=\rho \geqq \mu T^{-1}$, then $\rho\left(\operatorname{cl}\left(T\left(A^{*}\right)\right)\right)=1$. It follows that $\rho\left(B^{\prime}\right)=1$ and $T_{*}\left(W\left(A_{k}^{\prime}\right)\right) \subset W\left(B^{\prime}\right)$. Thus $T_{*}$ is continuous. Finally we note that if $\mathscr{L}_{1}$ and $\mathscr{L}_{2}$ are separating and disjunctive, then $T_{*} \mu_{x}=\nu_{T_{x}}$ where $\mu_{x} \in \operatorname{IR}\left(\mathscr{L}_{1}\right)$ is concentrated at $x$. Thus $T_{*}$ extends $T$.

We summarize these cases in the following theorem.

THEOREM 6.4. Let $\mathscr{L}_{1}$ and $\mathscr{L}_{2}$ be separating disjunctive lattices of $X$ and $Y$ respectively and $T: X \rightarrow Y$ be $\mathscr{L}_{1}-\mathscr{L}_{2}$ continuous.

(a) If $T: X \rightarrow Y$ is also a surjection such that $T^{-1}\left(\mathscr{L}_{2}\right)$ semiseparates $\mathscr{L}_{1}$, then the map $T^{*}: \operatorname{IR}\left(\mathscr{L}_{1}\right) \rightarrow \operatorname{IR}\left(\mathscr{L}_{2}\right)$ given by $T^{*}(\mu)=\mu T^{-1}$ is a continuous surjection extending $T$.

(b) If $T$ is also a surjection which is closed, the same conclusions as in (a) hold.

(c) If $\mathrm{cl}\left(T\left(\mathscr{L}_{1}\right)\right) \subset \mathscr{L}_{2}$ and $T$ satisfies condition (**) the map $T_{*}: \operatorname{IR}\left(\mathscr{L}_{1}\right) \rightarrow \operatorname{IR}\left(\mathscr{L}_{2}\right)$ given by $T_{*}(\mu)=\nu$ where $\nu$ is the unique 
element of $\operatorname{IR}\left(\mathscr{L}_{2}\right)$ which is $\geqq \mu T^{-1}$ on $\mathscr{L}_{2}$, is a continuous map extending $T$.

Proof. (a) and (c) have been given; (b) follows from Theorem 5.2, part (a).

REMARK 6.3. Condition (**) was first given by D. Harris in a topological setting with $\mathscr{L}_{1}$ and $\mathscr{L}_{2}$ the closed sets in $T_{1}$ topological spaces $X$ and $Y$ respectively. Such functions were called WO functions by him. However he did not demand that the original map be continuous. We have demanded $\mathscr{L}_{1}-\mathscr{L}_{2}$ continuity to show the relationship of $T_{*}$ to $\mu T^{-1}$. However we need not assume $T$ is $\mathscr{L}_{1}-\mathscr{L}_{2}$ continuous to define and obtain (c) of the theorem, namely: On the lattice generated by $\mathrm{cl}\left(T\left(\mathscr{L}_{1}\right)\right)$, we define the set function

$$
\rho\left(\operatorname{cl}\left(T A_{1}\right) \cap \cdots \cap \operatorname{cl}\left(T A_{n}\right)\right)=1
$$

if $\operatorname{cl}\left(T A_{1}\right) \cap \cdots \cap \operatorname{cl}\left(T A_{n}\right) \cap \operatorname{cl}(T B) \neq \varnothing$ for all $B \in \mathscr{L}_{1}$ with $\mu(B)=1$ and where $A_{1}, \cdots, A_{n} \in \mathscr{L}_{1}$, and 0 otherwise. Observe that if $\rho\left(\operatorname{cl}\left(T A_{1}\right) \cap \cdots \cap \operatorname{cl}\left(T A_{n}\right)\right)=1$, and $\rho\left(\operatorname{cl}\left(T B_{1}\right) \cap \cdots \cap \operatorname{cl}\left(T B_{m}\right)\right)=1$, $A_{1}, \cdots, A_{n}, B_{1}, \cdots, B_{m} \in \mathscr{L}_{1}$ then $\rho\left(\operatorname{cl}\left(T A_{1}\right) \cap \cdots \operatorname{cl}\left(T A_{n}\right) \cap \operatorname{cl}\left(T B_{1}\right) \cap\right.$ $\left.\cdots \cap \operatorname{cl}\left(T B_{m}\right)\right)=1$, for otherwise $\operatorname{cl}\left(T A_{1}\right) \cap \cdots \cap \operatorname{cl}\left(T A_{n}\right) \cap \operatorname{cl}\left(T B_{1}\right) \cap$ $\cdots \cap \operatorname{cl}\left(T B_{m}\right) \cap \operatorname{cl}(T B)=\varnothing$ for some $B \in \mathscr{L}_{1}$, with $\mu(B)=1$, but then $\left(\operatorname{cl}\left(T A_{1}\right)\right)^{\prime} \cup \cdots \cup\left(\operatorname{cl}\left(T A_{n}\right)\right)^{\prime} \cup\left(\operatorname{cl}\left(T B_{1}\right)\right)^{\prime} \cup \cdots \cup\left(\operatorname{cl}\left(T B_{n}\right)\right)^{\prime} \cup(\operatorname{cl}(T B))^{\prime}=Y$, and, there exists a $D \in \mathscr{L}_{1}, \mu(D)=1$, such that $\operatorname{cl}(T D) \subset \operatorname{cl}\left(T A_{i}\right)^{\prime}$ for some $i=1, \cdots, n$ or $\operatorname{cl}(T D) \subset \operatorname{cl}\left(T B_{i}\right)^{\prime}$, for some $i=1, \cdots, m$, or $\operatorname{cl}(T D) \subset \operatorname{cl}(T B)^{\prime}$. Clearly, the last alternative is not possible for this implies that $D \subset T^{-1}(\operatorname{cl}(T D)) \subset T^{-1}(\mathrm{cl}(T B))^{\prime}$ which implies that $D^{\prime} \supset B$, an impossibility. Therefore either $\operatorname{cl}(T D) \subset \operatorname{cl}\left(T A_{i}\right)^{\prime}$ for some $i=1, \cdots, n$, or $\operatorname{cl}(T D) \subset \operatorname{cl}\left(T B_{i}\right)^{\prime}$ for some $i=1, \cdots, m$, but both these lead to contradictions of the original assumptions that $\rho\left(\operatorname{cl}\left(T A_{1}\right) \cap \cdots \cap \operatorname{cl}\left(T A_{n}\right)\right)=1$ and $\rho\left(\operatorname{cl}\left(T B_{1}\right) \cap \cdots \cap \operatorname{cl}\left(T B_{n}\right)\right)=1$.

It is now readily seen, as before, that there exists a unique $\nu \in \operatorname{IR}\left(\mathscr{L}_{2}\right)$ such that $\rho \leqq \nu$ on the lattice generated by $\operatorname{cl}\left(T\left(\mathscr{L}_{1}\right)\right)$ and the remainder of the proof is unchanged.

It is a simple matter to give functorial characterizations of those matters analogous to those given by Harris and also to enlarge the class of extendable $T$ 's as in [25]. But we will not pursue these matters any further in this paper.

7. Subspaces. We will consider in this section subspace questions pertaining to repleteness which do not fall under the general mapping considerations of $\S \S 5$ and 6 . The techniques developed here seem to be particularly useful and have broad applications. We will assume throughout this section that $X$ is an abstract set and $\mathscr{L}$ is 
a lattice of subsets of $X$.

THEOREM 7.1. If $\mathscr{L}$ is a separating disjunctive delta lattice and if $X=\bigcup_{n=1}^{\infty} B_{n}$ where each $B_{n}$ is relatively $\mathscr{L}$-replete then $X$ is $\mathscr{L}$-replete.

Proof. Suppose $\mu \in I R(\sigma, \mathscr{L})$. We may assume without loss of generality that $\mu$ is defined on $\sigma(\mathscr{L}) . \mu(X)=1$ implies $\mu^{*}\left(B_{k}\right)=1$ for some $k$ where $\mu^{*}$ is the outer measure associated with $\mu$. Thus $B_{k}$ is thick in $X$ in the sense of [26] and we may project $\mu$ on $B_{k}$ by defining $\mu_{0}$ on $\sigma\left(\mathscr{L} \cap B_{k}\right)=\sigma(\mathscr{L}) \cap B_{k}$ as follows: $\mu_{0}\left(B \cap B_{k}\right)=\mu(B)$ where $B \in \sigma(\mathscr{L}) . \quad \mu_{0}$ is $\sigma$-smooth and $\mathscr{L} \cap B_{k}$-regular and its restriction to $\mathscr{A}\left(\mathscr{L} \cap B_{k}\right)$ is degenerate at some point of $p$ of $B_{k}$. Its unique extension to $\sigma\left(\mathscr{L} \cap B_{k}\right)$ is also degenerate at $p$. We need only show that $\mu$ is also concentrated at $p$. By $\mathscr{L}$-regularity it is sufficient to show that $\mu(L)=1$ if and only if $p \in L$ for any $L \in \mathscr{L}$. However this is immediate since $p \in L$ if and only if $p \in L \cap B_{k}$.

COROLLARY 7.1. If a Tychonoff space $X$ is the union of a sequence of $z$-embedded realcompact subspaces then $X$ is realcompact.

Proof. Take $\mathscr{L}$ in the theorem to be the zero sets of $X$.

COROLLARY 7.2. If a Tychonoff space $X$ is the union of a sequence of Borel replete subspaces then $X$ is Borel replete.

Proof. Take $\mathscr{L}$ in the theorem to be the Borel sets of $X$.

COROLlaRy 7.3 (see [23]). If $X$ is a zero set space and if $A \subset X$ is the union of a sequence of realcompact zero set spaces $A_{i}$, then $A$ is realcompact as a zero set space.

Proof. According to [23] $A_{i}$ is realcompact as a subspace if every $Z \cap A_{i}$ ultrafilter with the countable intersection property is fixed where $Z$ is the zero set structure of the original space. Thus the conditions of the theorem are built into Gordon's terminology.

(Note: Gordon calls a zero set space a set together with a strongly normal complement generated delta lattice.)

COROLlary 7.4 (Dykes). If a Tychonoff space is the union of a sequence of $\mathscr{F}$-replete (i.e., $\alpha$-complete) subspaces then $X$ is $\mathscr{F}$ replete.

Proof. Take $\mathscr{L}$ in the theorem to be the lattice of closed sets of $X$. 
Along these lines we have

COROLLARY 7.5. If a c.b. space is the union of a sequence of $\mathscr{F}$-replete (in particular Borel replete by (4.1)) subspaces, then $X$ is realcompact.

Proof. By the previous corollary $X$ is $\mathscr{F}$-replete; hence, by a result prior to $(4.5)$, realcompact.

REMARK 7.1. Corollary 7.1 includes Mrowka's result [43]: If a normal space $X$ is the union of a sequence of closed realcompact subspaces then $X$ is realcompact. Corollary 7.2 is a very strong improvement of Theorem 2.4 of [24].

Again, one may get further corollaries concerning $\mathscr{L}$-repleteness by combining these results with our previous mapping theorems and results in $\S 4$. The following theorems whose proofs are modifications of the above proof also have several applications.

THEOREM 7.2. If $\mathscr{L}$ is a separating and disjunctive delta lattice and if $X=\bigcup_{i=1}^{\infty} L_{i}^{\prime}$ where $L_{i} \in \mathscr{L}, i=1,2,3, \cdots$, where each $L_{i}^{\prime}$ is $\mathscr{L} \cap L_{i}^{\prime}$-almost replete, then $X$ is $\mathscr{L}$-almost replete.

Proof. Suppose $\mu \in I R\left(\mathscr{L}^{\prime}\right) \cap I\left(\sigma^{*}, \mathscr{L}\right)$. Then $\mu(X)=1$ implies $\mu\left(L_{k}^{\prime}\right)=1$ for some $k$ since $\mu$ is $\sigma$-smooth on $\mathscr{L}$. Define $\mu_{0}$ on $\mathscr{L}\left(\mathscr{L} \cap L_{k}^{\prime}\right)$ by $\mu_{0}\left(B_{0}\right)=\mu(B)$ where $B_{0}=B \cap L_{k}^{\prime}, B \in \mathscr{A}(\mathscr{L}) . \quad \mu_{0}$ is well defined since if $B_{0}=B_{1} \cap L_{k}^{\prime}=B_{2} \cap L_{k}^{\prime}$ where $B_{1}, B_{2} \in \mathscr{A}(\mathscr{L})$, then $B_{1} \triangle B_{2} \subset L_{k}$ where $B_{1} \triangle B_{2}$ is the symmetric difference of $B_{1}$ and $B_{2}$. This implies that $\mu\left(B_{1} \triangle B_{2}\right) \leqq \mu\left(L_{k}\right)=0$ and thus that $\mu\left(B_{1}\right)=\mu\left(B_{2}\right)$. It is easy to see that $\mu_{0} \in I R\left(\left(\mathscr{L} \cap L_{k}^{\prime}\right)^{\prime}\right)$. Furthermore $\mu$ is $\sigma$-smooth on $\mathscr{L} \cap L_{k}^{\prime}$. To see this note that if $B_{n} \cap L_{k}^{\prime} \downarrow \varnothing$, $n=1,2, \cdots$, then $B \cap L_{k}^{\prime}=\varnothing$ where $B=\bigcap_{i=1}^{\infty} B_{n}$. Thus $0=$ $\mu_{0}\left(B \cap L_{k}^{\prime}\right)=\mu(B)$. But then $\mu\left(B_{j}\right)=0$ for some $j$ (again since $\mu$ is $\sigma$-smooth on $\mathscr{L})$. Thus $\mu_{0}\left(B_{i} \cap L_{k}^{\prime}\right)=0$ for all $i \geqq j$. It follows that $\mu_{0}$ must be fixed at some point $p \in L_{k}^{\prime}$. A simple argument shows that $\mu$ is also fixed at $p$, and thus $X$ is $\mathscr{L}$ almost replete.

By an obvious modification of the proof we also obtain

THEOREM 7.3. If $X=\bigcup_{1}^{n} B_{k}$ where each $B_{k} \in \mathscr{A}(\mathscr{L})$ and each $B_{k}$ is $\mathscr{L} \cap B_{k}$-almost replete, where $\mathscr{L}$ is a separating disjunctive delta lattice then $X$ is $\mathscr{L}$-almost replete.

The following two corollaries improve both Theorems 4.2 and 4.1 respectively in [15]. 
COROLLARY 7.5. If a Tychonoff space is the union of a sequence of open almost realcompact subspaces, then $X$ is almost realcompact.

\section{Proof. Take $\mathscr{L}$ in Theorem 7.2 to be the lattice of closed sets of $X$.}

COROLLARY 7.6. If a Tychonoff space is the union of a finite number of almost realcompact subsets of $X$ belonging to $\mathscr{A}(\mathscr{F})$ then $X$ is almost realcompact.

\section{REFERENCES}

1. A. D. Alexandroff, Additive set functions in abstract spaces, Mat. Sb., (N. S) 8, 50 (1940), 307-348.

2. - Additive set functions in abstract spaces, Mat. Sb., (N. S.) 9, 51 (1941), $563-628$.

3. - Additive set functions in abstract spaces, Mat. Sb., (N. S.) 13, 55 (1943), 139-268.

4. R. Alo and H. L. Shapiro, Z-realcompactifications and normal bases, Australian J. Math., (11) (1970), 489-495.

5. - Normal Topological Spaces, Cambridge University Press, Cambridge, Mass., 1974.

6. G. Bachman and A. Sultan, Extensions of regular lattice measures with topological applications, J. Math. Anal. and Applications, (to appear).

7. G. Bachman and $\mathrm{R}$. Cohen, Regular lattice measures and repleteness, Comm. Pure Appl. Math., 26 (1973), 587-599.

8. G.Bachman, E. Beckenstein, L. Narici, and S. Warner, Rings of continuous functions with values in a topological field, Trans. Amer. Math. Soc., 204 (1975), 91-112.

9. B. Banaschewski, Uber nulldimensionale Raume, Math. Nach., 13 (1955), 129-140.

10. C. Carathéodory, Algebraic Theory of Measure and Integration, Chelsea Publ. Co., N. Y. 1963.

11. J. Chaber, Remarks on open-closed mappings, Fund. Math., 74 (1972), 197-208.

12. K. Chew, A characterization of $\mathrm{N}$-compact spaces, Proc. Amer. Math. Soc., 26 (1970), 679-682.

13. R. Cohen, Lattice measures and topologies, $\mathrm{Ph}$. D. Dissertation, Polytechnic Institute of New York, June 1974. (Also to appear in Ann. di Mat. Pura ed Applicata.)

14. C. Dowker and D. Papert Strauss, Separation axioms for frames, Colloquia Math. Soc. János Bolyai, Topics in Topology, Kessthaly (Hungary), (1972), 223-240.

15. N. Dykes, Generalizations of realcompact spaces, Pacific J. Math., $33(1970$, 571-581.

16. - Mappings and realcompact spaces, Pacific J. Math., 31 (1969), 347-358.

17. S. Fomin and S. Iliadis, The method of centered systems in the theory of topological spaces, Uspecki Mat. Nauk., 21 (1966), 47-76.

18. O. Frink, Compactifications and seminormal spaces, Amer. J. Math., 86 (1964), 602-607.

19. Z. Frolik, Prime filters with the C. I. P., Comm. Math. Univ. Carolinae, 13 (1972), $553-575$.

20. Applications of complete families of continuous functions to the theory of Q-spaces, Czech. Math. J., 11 (1961), 115-133.

21. - On almost realcompact spaces, Bull. Acad. Polon, Sci. Ser. Sci. Math. Astronom. Phys., 9 (1961), 247-250.

22. L. Gillman and M. Jerrison, Rings of Continuous Functions, University Series in 
Higher Mathematics, D. Van Nostrand, Princeton, N. J. (1960).

23. H. Gordon, Rings of functions determined by zero sets, Pacific J. Math., 36 (1971), 133-157.

24. A. Hager, G. Reynolds, M. Rice, Borel complete topological spaces, Fund. Math., 75 (1972), 135-143.

25. D. Hajek, Functions with continuous Wallman extensions, Czech. Math. J., 24 (1974), 40-43.

26. P. Halmos, Measure Theory, D. Van Nostrand, Toronto, New York, and London (1950).

27. J. Hardy and H. Lacey, Extensions of regular Borel measures, Pacific J. Math., 24 (1968), 271-282.

28. D. Haris, The Wallman compactification as a functor, General Topology and Its Applications, 1 (1971), 273-281.

29. H. Herrlich, E-kompacte Raume, Math. Zeit., 96 (1967), 228-255.

30. E. Hewitt, Rings of continuous functions I, Trans. Amer. Math. Soc., 64 (1948), 45-99.

31. T. Isiwata, Mappings and Spaces, Pacific J. Math., 20 (1967), 455-480.

32. D. Kappos, Probability Algebras and Stochastic Spaces, Academic Press, New York and London (1960).

33. J. Kelley, General Topology, Van Nostrand, Toronto, New York and London (1955). 34. J. Kelley, M. Nayak, T. Srinivasan, Premeasures on lattices of sets II, Vector and Operator Valued Measures and Applications, Academic Press, N. Y. and London, (1973) $155-164$.

35. A. Koltun, Lattice Measures and Compactifications, Ph. D. Dissertation, Polytechnic Institute of New York, (6/1975).

36. M. Levin and W. Stiles, On the regularity of measures in locally compact spaces, Proc. Amer. Math. Soc., 36 (1972), 201-6.

37. C. Liu, Absolutely closed spaces, Trans. Amer. Math. Soc., 130 (1968), 86-104.

38. C. Liu, The $\alpha$-closure $\alpha X$ of a topological space $X$, Proc. Amer. Math. Soc., 22 (1969), 620-624.

39. E. Marczewski, On compact measures, Fund. Math., 40 (1953), 113-124.

40. J. Marik, The Baire and Borel Measures, Czech. J. Math., 7 (1957), 248-253.

41. P. Meyer, Probability and Potentials, Blaisdell, Waltham, Toronto and London (1966).

42. W. Moran, Measures and mappings on topological spaces, Proc. London Math. Soc., 19 (1969), 493-508.

43. S. Mrowka, On the union of Q-spaces, Bull. Acad. Polon. Sci. Ser. Sci. Math. Astronom. Phys., 4 (1957), 365-368.

44. P. Nyikos, Not every O-dimensional realcompact space is $N$-compact, Bull. Amer. Math. Soc., 77 (1971), 392-396.

45. J. Olmstead, Lebesgue Theory on a Boolean Algebra, Trans. Amer. Math. Soc., 51 (1942), 164-193.

46. B. Pettis, On the extension of measures, Ann. of Math., 54 (1951), 186-197.

47. K. Ross and K. Stromberg, Baire sets and Baire Measures, Arkiv Mat., 6 (1965), 151-160.

48. R. Sikorski, The integral in a Boolean algebra, Coll. Math., 2 (1949), 20-26.

49. A. Sultan, Lattice Realcompactifications, Ann. di Mat. Pura ed Aplicata, Series 4, 106 (1975), 293-303.

50. F. Topsøe, Topology and measure, Springer Lecture Notes 133, Springer-Verlag, Berlin, Heidelberg and New York, 1970.

51. V. Varadarajan, Measures on topological spaces, Amer. Math. Soc. Translations, Series (2) 48 (1965), 161-228.

52. H. Wallman, Lattices and topological spaces, Ann. Math., 42 (1938), 687-697.

Received April 12, 1976.

Polytechnic Institute of New YoRk

AND

Queens College 



\section{PACIFIC JOURNAL OF MATHEMATICS}

\section{EDITORS}

RICHARD ARENS (Managing Editor)

University of California

Los Angeles, California 90024

\section{J. DugunduI}

Department of Mathematics University of Southern California Los Angeles, California 90007

D. Gilbarg and J. Milgram

Stanford University

Stanford, California 94305

\author{
R. A. Beaumont \\ University of Washington \\ Seattle, Washington 98105
}

\section{ASSOCIATE EDITORS}
B. H. NeUmanN
F. WOLF
K. YosHIDA

\section{SUPPORTING INSTITUTIONS}

\author{
UNIVERSITY OF BRITISH COLUMBIA \\ CALIFORNIA INSTITUTE OF TECHNOLOGY \\ UNIVERSITY OF CALIFORNIA \\ MONTANA STATE UNIVERSITY \\ UNIVERSITY OF NEVADA \\ NEW MEXICO STATE UNIVERSITY \\ OREGON STATE UNIVERSITY \\ UNIVERSITY OF OREGON \\ OSAKA UNIVERSITY
}

\author{
UNIVERSITY OF SOUTHERN CALIFORNIA \\ STANFORD UNIVERSITY \\ UNIVERSITY OF HAWAII \\ UNIVERSITY OF TOKYO \\ UNIVERSITY OF UTAH \\ WASHINGTON STATE UNIVERSITY \\ UNIVERSITY OF WASHINGTON \\ AMERICAN MATHEMATICAL SOCIETY
}

The Supporting Institutions listed above contribute to the cost of publication of this Journal, but they are not owners or publishers and have no responsibility for its content or policies.

Mathematical papers intended for publication in the Pacific Journal of Mathematics should be in typed form or offset-reproduced, (not dittoed), double spaced with large margins. Please do not use built up fractions in the text of your manuscript. You may however, use them in the displayed equations. Underline Greek letters in red, German in green, and script in blue. The first paragraph or two must be capable of being used separately as a synopsis of the entire paper. Items of the bibliography should not be cited there unless absolutely necessary, in which case they must be identified by author and Journal, rather than by item number. Manuscripts, in triplicate, may be sent to any one of the editors. Please classify according to the scheme of Math. Reviews, Index to Vol. 39. All other communications should be addressed to the managing editor, or Elaine Barth, University of California, Los Angeles, California, 90024.

The Pacific Journal of Mathematics expects the author's institution to pay page charges, and reserves the right to delay publication for nonpayment of charges in case of financial emergency.

100 reprints are provided free for each article, only if page charges have been substantially paid. Additional copies may be obtained at cost in multiples of 50 .

The Pacific Journal of Mathematics is issued monthly as of January 1966. Regular subscription rate: $\$ 72.00$ a year (6 Vols., 12 issues). Special rate: $\$ 36.00$ a year to individual members of supporting institutions.

Subscriptions, orders for back numbers, and changes of address should be sent to Pacific Journal of Mathematics, 103 Highland Boulevard, Berkeley, California, 94708.

PUBLISHED BY PACIFIC JOURNAL OF MATHEMATICS, A NON-PROFIT CORPORATION

Printed at Kokusai Bunken Insatsusha (International Academic Printing Co., Ltd.), 8-8, 3-chome, Takadanobaba, Shinjuku-ku, Tokyo 160, Japan. 


\section{Pacific Journal of Mathematics}

Patricia Andresen and Marvin David Marcus, Weyl's inequality and

quadratic forms on the Grassmannian .......................

George Bachman and Alan Sultan, Regular lattice measures: mappings and

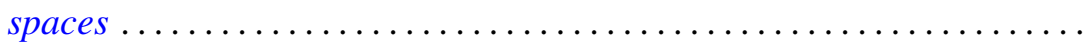

David Geoffrey Cantor, On certain algebraic integers and approximation by rational functions with integral coefficients ...................

James Richard Choike, On the value distribution of functions meromorphic in the unit disk with a spiral asymptotic value ..................

David Earl Dobbs, Divided rings and going-down................ 353

Mark Finkelstein and Robert James Whitley, Integrals of continuous

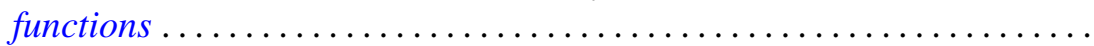

Ronald Owen Fulp and Joe Alton Marlin, Integrals of foliations on manifolds with a generalized symplectic structure ...............

Cheong Seng Hoo, Principal and induced fibrations .................

Wu-Chung Hsiang and Richard W. Sharpe, Parametrized surgery and

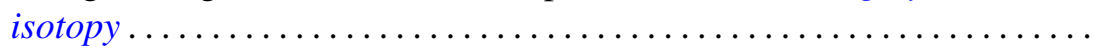

Surender Kumar Jain, Surjeet Singh and Robin Gregory Symonds, Rings whose proper cyclic modules are quasi-injective .................

Pushpa Juneja, On extreme points of the joint numerical range of commuting normal operators...

Athanassios G. Kartsatos, Nth order oscillations with middle terms of order $N-2$.

John Keith Luedeman, The generalized translational hull of a

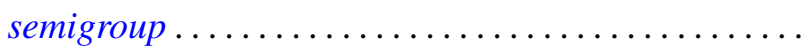

Louis Jackson Ratliff, Jr., The altitude formula and DVR's ...

Ralph Gordon Stanton, C. Sudler and Hugh C. Williams, An upper bound for the period of the simple continued fraction for $\sqrt{D}$...

David Westreich, Global analysis and periodic solutions of second order systems of nonlinear differential equations...

David Lee Armacost, Correction to: "Compactly cogenerated LCA

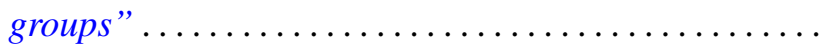

Jerry Malzan, Corrections to: "On groups with a single involution" .

David Westreich, Correction to: "Bifurcation of operator equations with unbounded linearized part" ...................... 